Article

\title{
New Classification of Collective Animal Behaviour as an Autonomous System
}

\author{
Takayuki Niizato ${ }^{1}, t, \ddagger *$, Kotaro Sakamoto ${ }^{2,}{ }^{2 *}$ and Yoh-ichi Mototake ${ }^{3}$, Hisashi Murakami ${ }^{4}$, \\ Takenori Tomaru ${ }^{5}$, Tomotaro Hoshika ${ }^{1}$, Toshiki Fukushima ${ }^{1}$ \\ Faculty of Engineering, Information and Systems University of Tsukuba, Japan \\ University of Tsukuba, Leading Graduate School Doctoral Program in Human Biology, Japan \\ The Institute of Statistical Mathematics, Tachikawa, Japan \\ University of Tokyo, Research Center for Advanced Science and Technology, Japan \\ Toyohashi University of Technology, Department of Computer Science and Engineering, Japan \\ * Correspondence: t_niizato@yahoo.co.jp, sakamoto@ccs.tsukuba.ac.jp \\ + Current address: Faculty of Engineering, Information and Systems University of Tsukuba, Japan \\ $\ddagger \quad$ These authors contributed equally to this work.
}

\begin{abstract}
Integrated information theory (IIT) was initially proposed to describe human consciousness in terms of intrinsic-causal brain network structures. This theory could potentially be used for conceptualising complex living systems. In a previous study, we analysed collective behaviour in Plecoglossus altivelis. We found that IIT 3.0 exhibits qualitative discontinuity between three and four schools of fish in terms of $\Phi$ values (i.e., group integrity). Other measures, such as mutual information, did not show such characteristics. In this study, we follow up on our previous findings and introduce two new factors. First, we define the global parameter settings to determine a different kind of group integrity. Second, we set several timescales (from $\Delta t=5 / 120 \mathrm{~s}$ to $\Delta t=120 / 120$ s). The results showed that we succeeded in classifying fish school according to their group size in terms of the degree of group integrity, despite the small group size. The concrete classification includes the followership for a two-fish school, fission-fusion for a three-fish school, emergence of leadership for a four-fish school, and emergence of Boid-like behaviour for a five-fish school. These minute classifications have never been revealed before. Finally, we discuss one of the longstanding paradoxes in collective behaviour, known as the heap paradox, for which two tentative answers could be provided through our IIT analysis.
\end{abstract}

Keywords: Integrated Information Theory; Collective Behaviour, Self-Organization; Cause and Effect Structure

\section{Introduction}

Global dynamic patterns in swarming [1-6], schooling (of fish) [7-11], flocking (of birds) [1217], genes [18], proteins [19], or neural networks [20-23], emerge from local interactions between self-organising individuals or components. Despite their complexity, these collective systems are capable of processing information efficiently in critical conditions, for example, individuals making a swift response [14-16] or a group making a good decision [24-26] in a changing environment. Individual conflicts do not necessarily disrupt group integrity and instead contribute to their collective response [27-29]. The complexity is characterised using statistical models and information theory. Self-organised criticality (SOC) [30] is considered as a convenient strategy that is evolutionarily adopted by living systems to efficiently cope with the external environment [31-35]. However, for smaller system sizes, heterogeneous interactions [36,37] result in different global motion patterns in $N=2$ 
and $N=3[38,39]$. Hence, some studies thoroughly investigated information transfer (or causal relationships) among individuals in small groups [40-50].

Most of the previous works on dynamical systems, including collective behaviours, focus on what the system 'does' — system's dynamical trajectory through its state space-that is, they take an extrinsic perspective on what is observed. Albantakis and Tononi shifted their focus towards a system's intrinsic/causal structure and considered what the system 'is' from its own intrinsic/causal perspective [51] based on the integrated information theory (IIT) [52,53]; they demonstrated that the intrinsic/causal complexity (as quantified by integrated information $\Phi$ ) correlates well with dynamical/statistical complexity. The integrated information, represented by $\Phi$, is defined as a degree of information loss (or an increase in uncertainty [54]) caused by the removal of certain edges of the Bayesian network [55-62]. The metric was successful in discriminating not only various states of lost consciousness, such as dreamless sleep [63], general anaesthesia [64], or vegetative states [65] but also the different states of complex systems, such as the generalised Ising model [66], coupled oscillators [67], and coupled mapping [68].

The aim of IIT 3.0, as we mentioned earlier, is to try to shift the paradigm from 'what the system does' to 'what the system is' [51,60,61,69-71]. The former tries to analyse the system based on its external behaviour, whereas the latter tries to analyse the system according to its intrinsic causal structure. This difference is elegantly captured by the analysis on the cellular automata. The cellular automaton refers to the time-development system defined by binary states with the application of simple rules. Despite its simple construction, it has rich connotations. In general, a cell's behaviour can be classified into four classes: Class I (uniform stable state), Class II (periodic or non-uniform stable state), Class III (chaos), and Class IV (complex system as the universal computation or edge of the chaos) [72,73]. In the past decades, almost all studies on the cellular automaton focused on their behavioural differences $[45,45,74,75]$, although some exceptions exist [76]. The IIT 3.0 application of Albantakis et al. [51] succeeded in classifying these four patterns without referring to the external cell behaviour ('what the system does'). Their analysis was based on a small number of cells (up to six cells), which is insufficient to show the behavioural difference among the rules. Therefore, there is no room for the external behavioural analysis here. Furthermore, Albantakis et al. also pointed out the possibility of the existence of an unobserved new class from the intrinsic causal cell structure ('what the system is').

The application of IIT 3.0 to a system has at least two important implications. First, IIT 3.0 can select some characteristics of the external cell behaviour from their intrinsic causal structure. As such, IIT 3.0 can classify small systems in terms of autonomous systems, which are not distinguished from their external behaviours. This was partly achieved in our previous study [77], in which we applied IIT 3.0 to schools of 2-5 fish (Plecoglossus altivelis) and found that $\Phi$ was discontinuous between three and four schools of fish in terms of the emergence of leadership. We also showed that this kind of leadership must be strictly discriminated from the conventional leadership, such as that defined by the transfer entropy. Indeed, IIT-induced leadership is never replicated using other measures (i.e., mutual information and the sum of the transfer entropy) and under some artificial conditions (Boid and homogeneous Markov models). The critical point is that the leadership, which usually is inferred from the external behaviour, can make detections from the causal structure of cells without referring to their external behaviour.

In the present study, we extended our previous approach to examine how integrated information changes under different timescales by adding new parameter settings as the global parameter settings. By applying these metrics, we succeeded in classifying the different sizes of schools as qualitatively different autonomous systems (e.g., followership, fission-fusion, leadership, and Boid-like interaction). This is a novel type of classification of collective animal behaviour. Finally, we discuss the heap paradox on collective behaviour, for which we show that our IIT analysis can provide two kinds of tentative answers. 


\section{Results}

\subsection{Brief summary of IIT 3.0}

First, we provide a brief summary of IIT 3.0. The IIT models a system, $S$, using the discrete time multivariate stochastic process composed of $N$ interacting components as follows:

$$
p\left(X_{0}, X_{\Delta t}, \ldots, X_{t}, X_{t+\Delta t}, \ldots, X_{T}\right),
$$

which satisfies the Markov property,

$$
p\left(X_{0}, X_{\Delta t}, \ldots, X_{t}, X_{t+\Delta t}, \ldots, X_{T}\right)=p\left(X_{0}\right) \prod_{t=\Delta t}^{T} p\left(X_{t} \mid X_{t-\Delta t}\right) .
$$

Such a discrete Markovian dynamical system, $S$, is defined using a directed graph of interconnected nodes (in this study, we assumed a complete graph) and its TPM, which specifies the conditional probability distribution, $p\left(X_{t} \mid X_{t-\Delta t}\right)$. Each state vector, $X_{t}$, comprises binary variables $x_{t_{i}}$, where $i=1,2, \ldots, n(n \in N)$.

A joint distribution, $p_{\text {cause-effect }}$, is defined as

$$
p_{\text {cause-effect }}\left(X_{t-\Delta t}, X_{t}\right):=p_{u}\left(X_{t-\Delta t}\right) p_{\text {effect }}\left(X_{t} \mid X_{t-\Delta t}\right) \text {. }
$$

The marginal distribution, $p_{u}\left(X_{t-\Delta t}\right)$, is a uniform distribution, which provides the maximum entropy distribution.

From the aforementioned joint probability, the backward transitional probability distribution can be defined as follows:

$$
p_{\text {effect }}\left(X_{t-\Delta t} \mid X_{t}\right):=\frac{p_{\text {cause-effect }}\left(X_{t-\Delta t}, X_{t}\right)}{\sum_{X_{t-\Delta t}} p_{\text {cause-effect }}\left(X_{t-\Delta t}, X_{t}\right)^{\prime}}
$$

and the forward transitional probability distribution is defined as follows:

$$
p_{\text {cause }}\left(X_{t} \mid X_{t-\Delta t}\right):=p\left(X_{t} \mid X_{t-\Delta t}\right) \text {. }
$$

These are constructed and referred to as the cause repertoire and the effect repertoire of state $X_{t}$, respectively, which are calculated for a set of nodes within the subsystem, for mechanism $M \subseteq S$ over another set of nodes within the subsystem, or for a purview of the mechanism.

After evaluating the information of the mechanism over a purview, we consider the integrated information, $\varphi_{\text {cause-effect, }}$ of a set of system elements at state $X$. The integrated information can be defined as follows:

$$
\begin{aligned}
& \phi_{\text {cause-effect }}:=\min \left(\phi_{\text {effect }}, \phi_{\text {cause }}\right) \\
& \phi_{\text {effect }}:=\min _{i \in I} D\left(p_{\text {effect }} \| p_{\text {effect }}^{(i)}\right) \\
& \phi_{\text {cause }}:=\min _{i \in I} D\left(p_{\text {cause }} \| p_{\text {cause }}^{(i)}\right),
\end{aligned}
$$

where the system is decomposed in all possible ways into $I$.

Integrated information $\varphi$ is estimated by quantifying the extent to which the cause and effect repertoires of the mechanism-purview pair can be reduced to the repertoires of its components. The amount of irreducibility of a mechanism over a purview with respect to a partition is quantified as the divergence between unpartitioned repertoire $p$ and partitioned repertoire $p^{(i)}$. The partition that yields 
the minimum irreducibility is called the minimum-information partition (MIP). Integrated information $\varphi$ of a mechanism-purview pair is defined as the divergence between the unpartitioned repertoire and repertoires partitioned by the MIP. The maximum $\varphi$ value is then determined for overall possible purviews to determine the maximally irreducible cause and maximally irreducible effect specified by a mechanism.

$$
\varphi_{\text {cause }}^{\max }:=\max _{j \in C}\left(\varphi_{\text {cause }}^{j}\right), \varphi_{\text {effect }}^{\max }:=\max _{j \in C}\left(\varphi_{\text {effect }}^{j}\right),
$$

where $C=2^{N}-1$. (In this study, we adopted the 'cut one' approximation that only evaluates $2 N$ bipartitions, thereby severing the edges of a node to the remaining network.)

The $\varphi$ value of the concept as a whole or the maximally integrated cause-effect information is the minimum of the maximally integrated cause information, $\varphi_{\text {cause, }}$ and maximally integrated effect information, $\varphi_{\text {effect }}$.

$$
\varphi_{\text {cause-effect }}^{\max }:=\min \left(\varphi_{\text {cause, }}^{\max } \varphi_{\text {effect }}^{\max }\right)
$$

If the mechanism's maximally irreducible cause has $\varphi_{\text {cause }}>0$ and its maximally irreducible effect has $\varphi_{\text {effect }}>0$ (equivalently, $\varphi_{\text {cause-effect }}^{\max }>0$ ), the mechanism is said to specify a concept.

A maximally irreducible cause-effect structure composed of concepts is called a maximally irreducible conceptual structure (MICS), or conceptual structure (constellation of concepts) $C(S)$ (the set of all concepts specified by the subsystem characterising all the causal constraints intrinsic to the physical system) by simply iterating the computation of the concepts over all the mechanisms, $M \in \mathcal{P}(\mathcal{S})$, where $\mathcal{P}(\mathcal{S})$ is the power set of the subsystem nodes.

Integrated conceptual information, $\Phi$ (also known as big phi, a measure of the system's strong/integration irreducibility), is estimated by partitioning the set of elements into subsets with unidirectional cuts. The unidirectional bipartitions, $P_{\rightarrow}=\left\{S^{(1)} ; S^{(2)}\right\}$, of physical system $S$ are obtained by partitioning the subsystem into two parts, namely, $S^{(1)}$ and $S^{(2)}$ and cutting the edges from these parts (the connections are substituted with noise). We then calculate the conceptual structure of the partitioned system, $C\left(S^{P \rightarrow}\right)$, and compare it with $C(S)$ to evaluate the difference caused by partition. An MIP (a search over all possible directed partitions) is then performed to identify the partition that causes the least amount of difference to the conceptual structure. $\Phi$ measures the irreducibility of a conceptual structure by quantifying the difference created by the MIP to the concepts and their $\varphi$ values of the system.

$$
\Phi=\min _{P \rightarrow} D\left(C(S), C\left(S^{P \rightarrow}\right)\right)
$$

Difference $D$ between the two conceptual structures was evaluated by an extended version of the earth mover's distance, which is the cost of transforming a conceptual structure, $C(S)$, into another $C\left(S^{P} \rightarrow\right)$ in a concept space. The candidate subset with the largest $\Phi$ is called the main complex of the system $S$.

\subsection{Definition of the local parameter settings}

To apply IIT (especially, IIT 3.0) to schools (of fish), it is necessary to first determine their binary states because they do not possess a specific firing state such as neurons in the brain. In our previous study, we proposed the definitions of binary states for each parameter as a threshold for ON states [77].

In this study, we provide a summary of the two types of parameter settings (for the detailed mathematical definition, refer to Materials and Methods). First, the set of local parameters is composed of three types of interactions: a distance (i.e., contacting other individuals), field view (i.e., observing other individuals using one's sight), and turning rate (i.e., state of being affected by other individual's movements). These three parameters reflect the local interactions, such as Boids, which are often observed in various flocking models. Second, the set of global parameters is composed of two types of 
interactions: distance from the centre of mass and the difference from the averaged group direction (explained in detail in section 2.2.1. Here, we focus on only the local parameter settings.

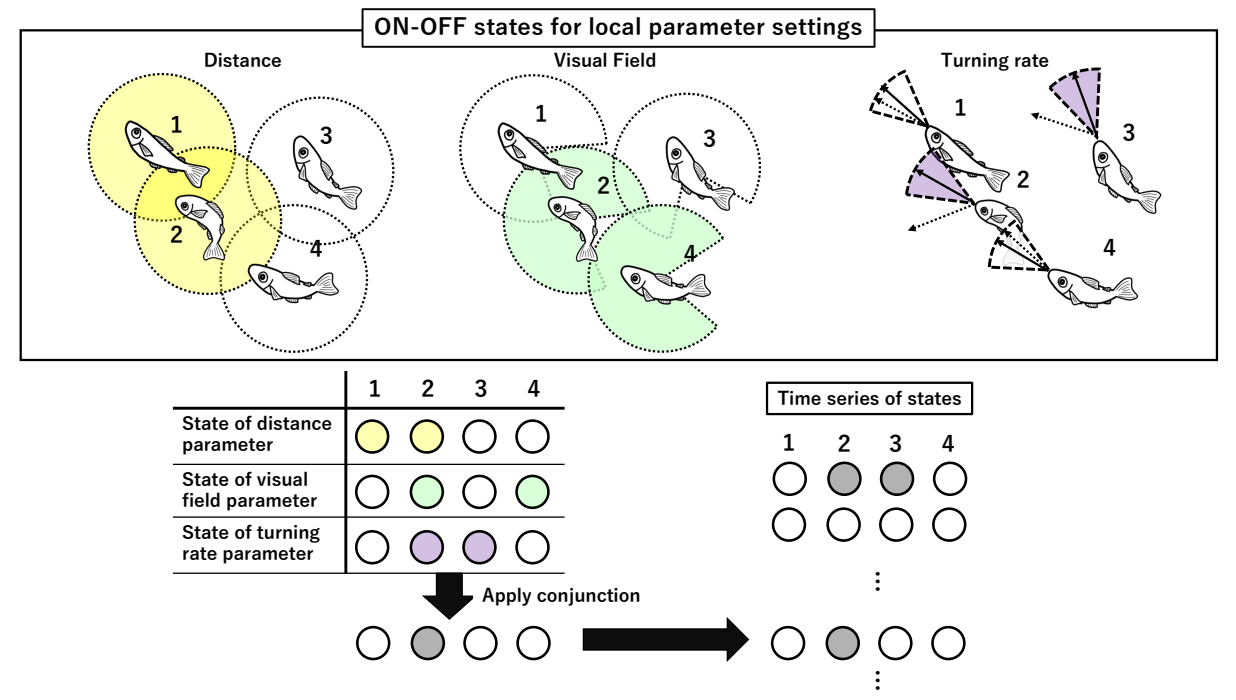

(a) The definition of $\mathrm{ON}$ and OFF states for local parameter settings.

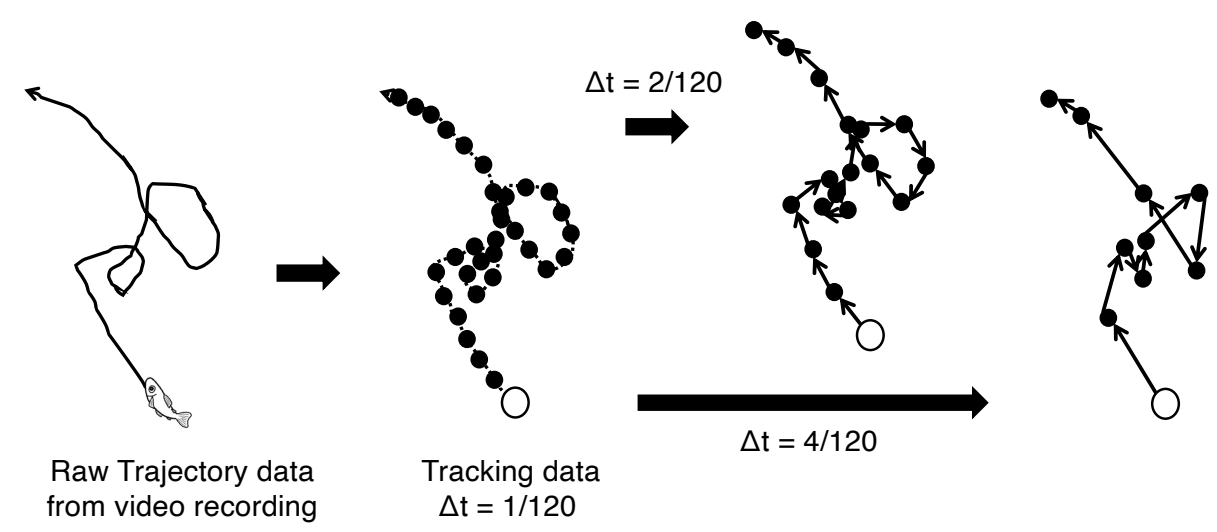

(b) Timescale rules for the analysis.

Figure 1. (a) Definition of ON and OFF states for local parameter settings. Three parameters are used to determine a school's state (Yellow: distance, Blue: visual field, Purple: turning rate). The coloured individuals are in the $\mathrm{ON}$ state. We take a conjunction of the three school states to obtain the final school state at time $t$. Subsequently, we compute $\Phi$ from a time series of these states using PyPhi. (b) Timescale rules. The video recordings present each trajectory. The frame rate of our device is $120 \mathrm{fps}$. The velocity vectors of the time scale, $\Delta t$, define every $\Delta t$ step-length when the time scale is $\Delta t=\delta t / 120$. The timescales are $\Delta t=5 / 120, \Delta t=10 / 120, \Delta t=20 / 120, \Delta t=40 / 120, \Delta t=80 / 120$, and $\Delta t=120 / 120 \mathrm{~s}$. Large time scales eliminate subtle noise-like movements in a school (or support the predictability of other fish's movements).

After obtaining a binary state of those parameter settings for each individual, we apply conjunction (or the AND operator) to the three bits of information as collective states at time $t$. For example, if the states of distance, visual field, and turning rate are ON, OFF, and OFF, respectively, for fish $i$, then $i$ is in the OFF state. The application of the same procedure to all the $N$-fishes in the group gives a collective state at time $t$. Note that the central interest of this study is not an individual's perceptual abilities (e.g., the fixed visual field) but the individual's effective perceptions under the dynamical interactions as parameter settings. More precisely, the interaction radius and visual field should be interpreted as the "effective" interaction radius and the "effective" visual field, respectively [78]. 

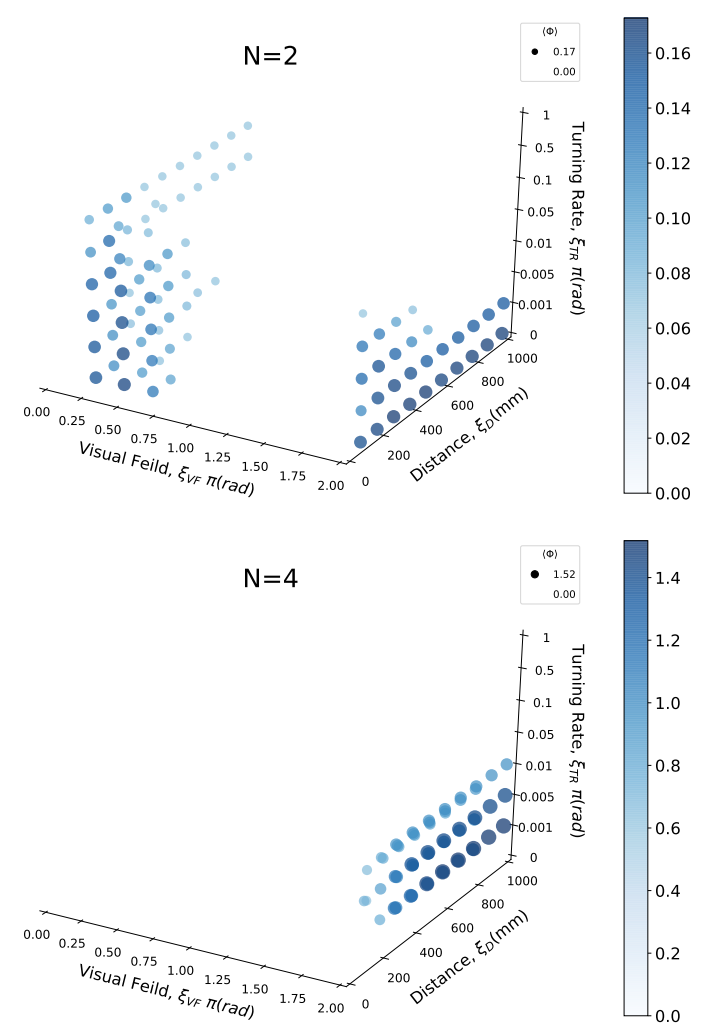

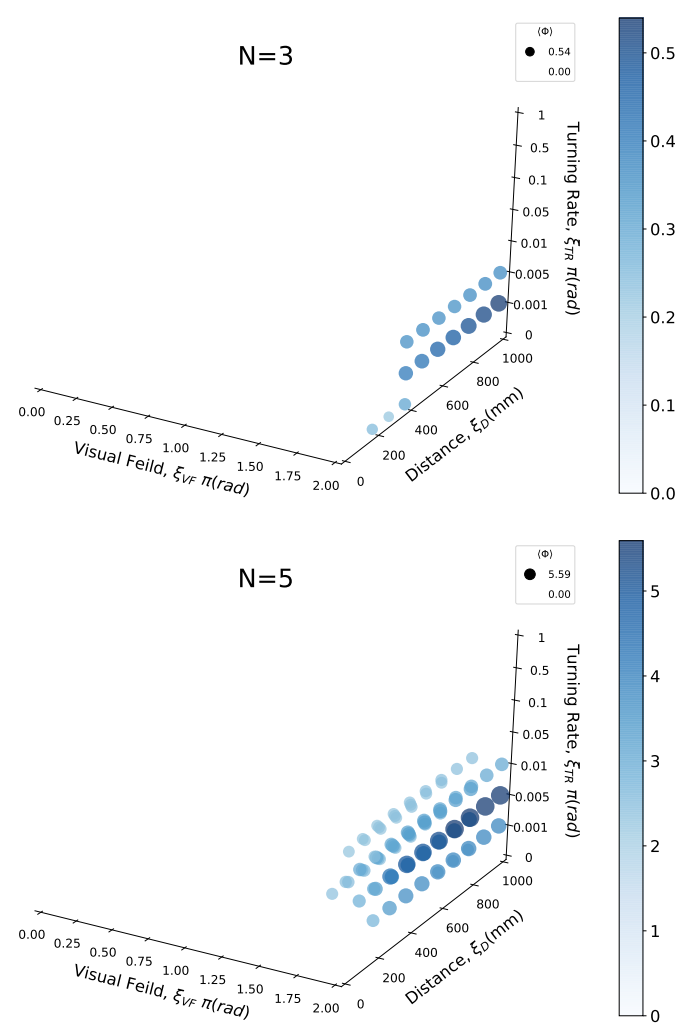

Figure 2. 3D distribution of the mean $\langle\Phi(N)\rangle$ values with respect to the three parameters according to the group size for all experimental data $(\Delta t=20 / 120 \mathrm{~s})$. The ball size and shaded colours represent the $\langle\Phi(N)\rangle$ strength. For the sake of visibility, we only show the points over $0.4^{*}\langle\Phi(N)\rangle_{M A X}$, where $\langle\Phi(N)\rangle_{\text {MAX }}$ implies the $\langle\Phi(N)\rangle$ values of the maximum cell for each group size. We measured the $\langle\Phi(N)\rangle$ values over the main complexes and full subsystems throughout our analysis. All the graphs refer only to the main complexes. For similar distributions, please refer to [77]. Figures S1 and S2 include all the mean $\langle\Phi(N)\rangle$ and mean $\sigma^{2}(\Phi(N))$ values for all the timescales.

The application of conjunction has critical biological implications because an analogy from the Boids model, referred by several researchers, was adopted in a collective animal behaviour as collective states [32,79-83]. Almost every interaction in the general Boids model adopts the interaction radius with blind spots, adding noise to the agent's turning directions. The conjunction of the above-mentioned three parameters replicates this statement; it would lose meaning if we replace the AND operator with the OR operator. For instance, when the two parameters (distance and visual field) are combined with AND, and the remaining (turning rate) are combined with OR [i.e., OR(AND(Distance, Visual Field), Turning parameter)], the system allows the ON states irrespective of the states of either of the two parameters (distance or visual field). This example violates the notion of the interaction distance with a blind spot.

In this study, the timescale has several implications in our analysis. In the timescale, the position vectors for every $\Delta t$ step of each fish are considered (Figure 1(b)). The larger the timescale, the more predictable and regular are the trajectories in this setting. In our previous study, we restricted our primary focus to a very short timescale $(\Delta t=5 / 120 \mathrm{~s})$ and more narrowly ranged parameter setting (for the distance and visual field parameters only). A larger amount of parameter settings reveal more precise and minute causal structures, as shown later in text. The transition probability matrix (TPM), which is assumed to have conditional independence [57,62], is obtained from the entire sequence of the collective states (Figure 1(a)). The PyPhi [70] computes all the IIT related values by using this TPM. These bit sequences as collective states are not only sensitive to parameter selection but also to timescale selection, i.e., the length of the time step. In this study, we examined a vast range of timescales, such as $\Delta t=5 / 120,10 / 120,20 / 120,40 / 120,80 / 120$, and $120 / 120$ s (see 4 ). 
In addition to the TPM, the network structure of the group must also compute IIT-related values. Therefore, we set the network structure of the school (of fish) as all connected network without self-loops. This is because the minimum distance between any pair of fish is less than $5 \mathrm{~mm}$ in our experiment (see 4). It would be sufficient to suppose that some type of interaction occurs amongst all the fishes by mutual contact throughout a series of an event (i.e., roughly 10-15 min of free swimming). We chose this network structure in this study because according to the results of our previous study, for the network structure including a self-loop, the MIP cut shows weak correspondence to the leadership in the group compared with a non-self-loop network [77]. The self-loop, when included in a network, reflects the past histories of each movement. Considering that the past movements to the network may fail to determine the pure inter-relations among fishes in the group, the fully connected structure without a self-loop is the most suitable structure for the IIT analysis.

Note that each collective state has a unique corresponding computed $\Phi$ value in the above-mentioned procedure. For example, in the case of a two-fish school, in which all collective states are $(0,0),(1,0),(0,1)$, and $(1,1)$, we have four values of $\Phi$, that is, $\Phi_{00}, \Phi_{10}, \Phi_{01}$, and $\Phi_{11}$, respectively. In this study, we mainly focused on the average $\Phi$ values (denoted as $\langle\Phi(N)\rangle$ ) for all collective states ( $2^{N}$ for $N$ fish schools). We also used $\sigma(\Phi(N))^{2}$ as variance of all $2^{N} \Phi$ values.

\subsection{1. $\Phi$ values for local parameter settings across timescales}

Figure 2 shows the example of $\langle\Phi(N)\rangle$ distributions for the local parameter settings when the timescale is $\Delta t=20 / 120 \mathrm{~s}$. The ball size and gradation of the blue colour represent the $\langle\Phi(N)\rangle$ values for each cell. We omitted the small $\langle\Phi(N)\rangle$ values under specific values (Figure 2) from the graph for the sake of their visibility. The dense location of $\langle\Phi(N)\rangle$ is meaningful for our analysis because the $\langle\Phi(N)\rangle$ values represent the group's integrity in the school (of fish). According to the definition of IIT 3.0, the more the system of interest obtains the feedback information structure (i.e., mutually connected with each other), the more that system has high $\langle\Phi(N)\rangle$ values. In contrast, the areas with low $\langle\Phi(N)\rangle$ values correspond to few feedback structures (only feedforward or mutual independent) as an autonomous system.

Before detailing our analysis, let us make note of some of the remarkable features that can be observed in Figure 2. First, the distribution of a school of two fishes differs from other distributions. Large $\langle\Phi(N)\rangle$ values are concentrated on a low visual-field area ( $\left.\xi_{V F}<\pi / 2\right)$ in a two-fish school. In three- to five-fish schools, the $\langle\Phi(N)\rangle$ distribution tends to be concentrated around a high $\xi_{V F}$ area. In our previous study, under particular conditions (especially, in the region of $\pi / 2<\xi_{V F}<\pi$ ), we confirmed the emergence of a leadership relationship. A large $\xi_{T R}$ value did not contribute to group integrity for any of the group sizes. In high $\xi_{T R}$ regions of more than 0.05 rad, no high $\langle\Phi(N)\rangle$ values were confirmed. This tendency has two implications: signalling only the intense direction changes (high $\xi_{T R}$ value) is not helpful for group integration, and the ON/OFF states are not symmetric owing to the combination of other parameters. If the relation is symmetrical, $\langle\Phi(N)\rangle$ values of the lower and higher $\xi_{T R}$ regions would show the same distribution; this is not true. The interpretation of ON/OFF could differ according to the given conditions. The latter will be discussed in "followership" relation to a school of two fish. 
(a)

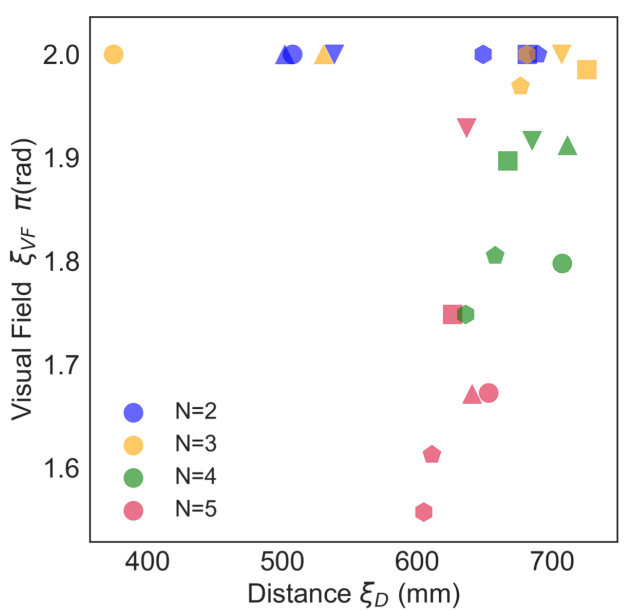

(b)

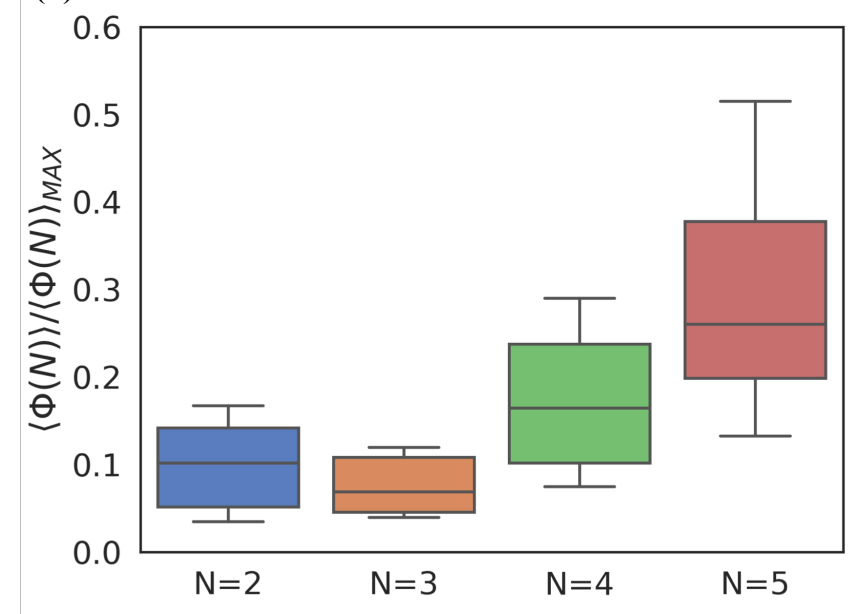

Figure 3. (a) The mean distribution over $\frac{1}{2}\langle\Phi(N)\rangle_{M A X}$, where $\xi_{V F}>\pi$ and $\xi_{T R}<.05$. Each colour corresponds to the group size, and each shape corresponds to the time scale: $\Delta t=5 / 120 \mathrm{~s}$ (circle), 10/120 s (upward triangle), 20/120 s (downward triangle), 40/120 s (rectangle), 80/120 s (pentagon), and $120 / 120 \mathrm{~s}$ (hexagon). $\langle\Phi(N)\rangle_{\text {MAX }}$ represents the maximum $\langle\Phi(N)\rangle$ value of all the cells in the $\langle\Phi(N)\rangle$ distribution. The distributions of $N=2$ and $N=3$ for all the scales concentrated on the complete visual fields $\left(\xi_{V F}=2 \pi\right)$. In contrast, the distributions of $N=4$ and $N=5$ for all the scales concentrated on the lower right field $\left(\xi_{V F}<2 \pi\right)$. (b) The box plot for the mean normalised $\langle\Phi(N)\rangle$ values, where $\pi<\xi_{V F}<2 \pi$ for all datasets. Each datum is divided by the $\langle\Phi(N)\rangle_{M A X}$ in the region of $\pi<\xi_{V F}<2 \pi$ and $\xi_{V F}<p i$ (note this graph uses all the $\langle\Phi(N)\rangle$ values: no restriction such as $\left.\frac{1}{2}\langle\Phi(N)\rangle_{\text {MAX }}\right)$. The $\langle\Phi(N)\rangle$ values of four- and five-fish schools are significantly higher than those of two- and three-fish schools. For comparison, Figure S3 presents the same box plot for high turning rates of $\xi_{T R} \geq 0.05$.

\subsubsection{Leadership in the four- and five-fish schools}

As the examination and comparison of all the scales and parameter settings are difficult, we divided some regions in the parameter space for convenience. First, we divided the regions with $\xi_{T R}<0.05$ and $\xi_{T R} \geq 0.05$ because the $\langle\Phi(N)\rangle$ values are very low in the high $\xi_{T R}$ regions $(\geq 0.05)$. The difference in the $\langle\Phi(N)\rangle$ distribution is mainly concentrated on the low $\xi_{T R}$ regions (Figure 2; the overall results are shown in Figures S1 and S2.)

Figure 3 (a) presents one of the main essences in the local parameter settings. Each point in Figure 3 (a) represents the weighted average position at which the $x$-axis represents the distance $\left(\xi_{D}\right)$ and the $y$-axis represents the visual field $\left(\xi_{V F}\right)$. We can visually divide the distributions into two groups: $N=2,3$ and $N=4,5$. We call this discontinuity in the $\langle\Phi(N)\rangle$ distributions between three- and four-fish schools as "the emergence of leadership" because the mean $\langle\Phi(N)\rangle$ values with blind spots (i.e., $\xi_{V F} \neq 2 \pi$ ) in the school are high only when the group size is more than four (Figure 3 (ii); the statistical tests presented in Table S1: only two- and three-fish schools show no statistical difference).

We define "leadership" in the group satisfying the following two properties: (i) the positional leader (the head position along the average group direction) and (ii) the asymmetric information lies between the leader and its followers. In fact, our previous analysis found that the head along the average group direction (property (i)) is strongly correlated with MIP cuts, especially in a fully connected network without self-loops [77]. This MIP cut position indicates a feedforward relationship between the leader and its followers because the MIP cut is performed unidirectionally; this is selected as the weakest link in the network structure (property (ii)). In other words, the opposite information flows are always larger than the first cut. Indeed, Figure 3 (ii) supports our consideration. The average $\langle\Phi(N)\rangle$ value in $\pi / 2<\xi_{V F}<\pi$ regions increases when the group size surpasses four (satisfies property (ii)). Furthermore, IIT-induced leadership (i.e., corresponding to MIP cut) highly correlates 
with the positional leadership [77]. Therefore, the groups with over four-fish schools satisfy all of the leadership conditions.

(a)

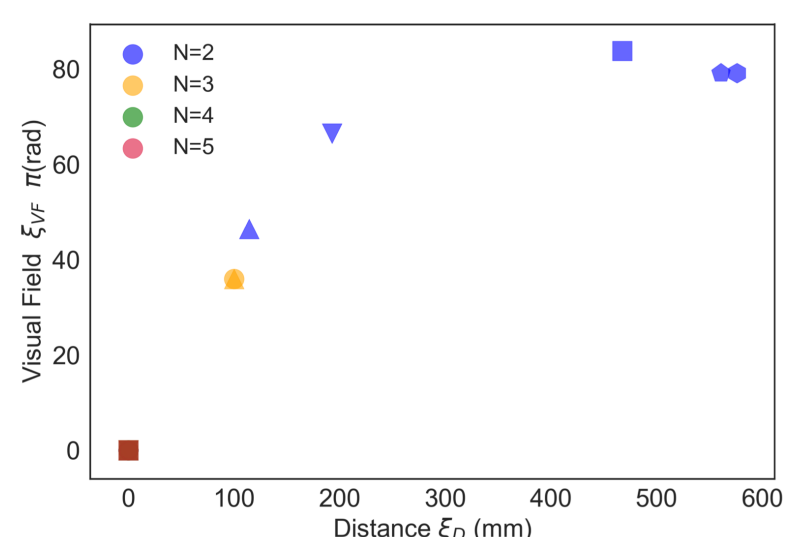

(b)

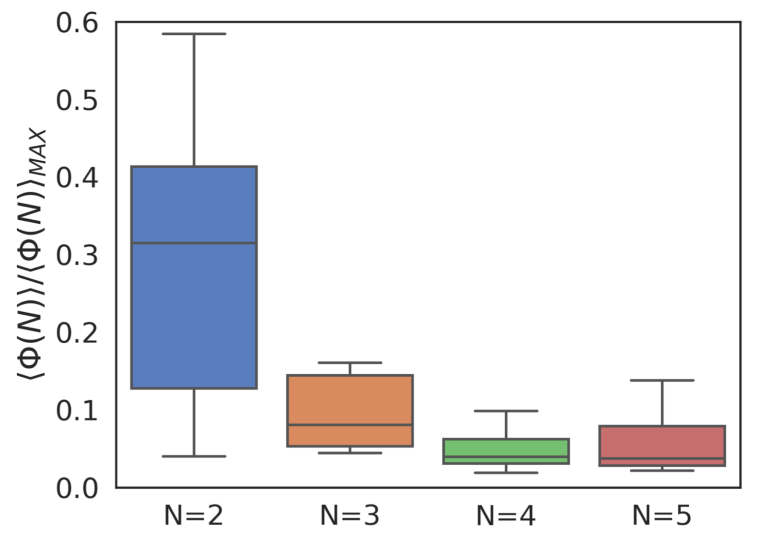

Figure 4. (a) The mean distribution over $\frac{1}{2}\langle\Phi(N)\rangle_{M A X}$, where $\xi_{V F}<\pi$ and $\xi_{T R}<0.05$. Each colour corresponds to the group size, and each shape corresponds to the time scale: $\Delta t=5 / 120 \mathrm{~s}$ (circle), 10/120 s (upward triangle), 20/120 s (downward triangle), 40/120 s (rectangle), 80/120 s (pentagon), and $120 / 120 \mathrm{~s}$ (hexagon). No value exceeds $\frac{1}{2}\langle\Phi(N)\rangle_{\text {MAX }}$ for the four- and five-fish schools. Some of the three-fish school exceed this value; however, only in a few samples. (b) The box plot for the mean normalised $\Phi$ values, where $0<\xi_{T R}<\pi$ for all datasets. The data were divided with the maximum $\langle\Phi(N)\rangle_{M A X}$ in $0<\xi_{V F}<\pi$ and $\xi_{T R}<0.05$ (note this graph uses all the $\langle\Phi(N)\rangle$ values: no restriction such as $\left.\frac{1}{2}\langle\Phi(N)\rangle_{M A X}\right)$. The $\langle\Phi(N)\rangle$ values in the two-fish school were determined as "followership", which is the opposite of "leadership". For comparison, Figure S4 depicts the box plot for the high turning rate, $\xi_{T R} \geq 0.05$, under the same condition. The statistical test is included in Table S1.

\subsubsection{Followership in the school of two fishes}

Next, we focus on the $0<\xi_{V F}<\pi$ regions. In these regions, only the schools of two fishes show distributions for high $\langle\Phi(N)\rangle$ values. The parameter distributions in Figure 4 (a) show a relative contrast with the same distributions depicted in Figure 3 (a). Only two-fish schools show high $\langle\Phi(N)\rangle$ values over $0.4^{*}\langle\Phi(N)\rangle_{M A X}$; in contrast, no large $\langle\Phi(N)\rangle$ values were observed in over four-fish schools.

This visual consideration can be confirmed in Figure 4 (b). We normalised all the $\langle\Phi(N)\rangle$ values using $\langle\Phi(N)\rangle_{\text {MAX }}$ for each group size. The mean normalised $\Phi$ value of $N=2$ (the mean $\langle\Phi(N=$ $2)\rangle \simeq 0.29$ ) in this region (Figure $4(\mathrm{~b}))$ is as high as the that in $N=5$ (the mean $\langle\Phi(N=5)\rangle \simeq 0.27$; Figure $3(\mathrm{~b}))$. We found that only the mean $\langle\Phi(N)\rangle$ in $\xi_{V F}$ lower regions showed significant difference to those of the remaining group (see Table S1). Therefore, the high integrity of this lower $\xi_{V F}$ in the region of two-fish schools suggests the existence of important properties in terms of group integrity.

We refer to this tendency, which is only observed in the school of two fishes, as "followership" owing to the following reasons. First, the narrow regions of $\xi_{V F}$ parameters correspond to large $\langle\Phi(N)\rangle$ values in the school of two fishes. Compared with the leadership in four- and five-fish schools, the narrower $\xi_{V F}$ value in a two-fish school shows the tracking of the leader to avoid losing sight of the leader. This leader always positions ahead of the follower by definition. Second, the MIP cut point also functions as asymmetric information processing between the leader and its follower. As the MIP cut in the school of two fishes functions exclusively to each other, the asymmetric information flow always exists between the two fishes.

The above-mentioned fact seems to pass all of the leadership conditions; however, there is a notable difference between the leadership and followership. The reversed relation never increases the $\Phi$ values of the two-fish school as that in the low $\xi_{V F}$ regions. If the role between the leader and followers is commutable (symmetric ON/OFF relation), the same high $\langle\Phi(N)\rangle$ values in the two-fish 
school must also be observed in high $\xi_{V F}$ regions; however, this is not the case for two-fish schools (Figure 3 (b) $N=2$ ). Therefore, we can discriminate the concepts of "followership" and "leadership" in this study.

\section{3. $\Phi$ values for global parameter settings across timescales}
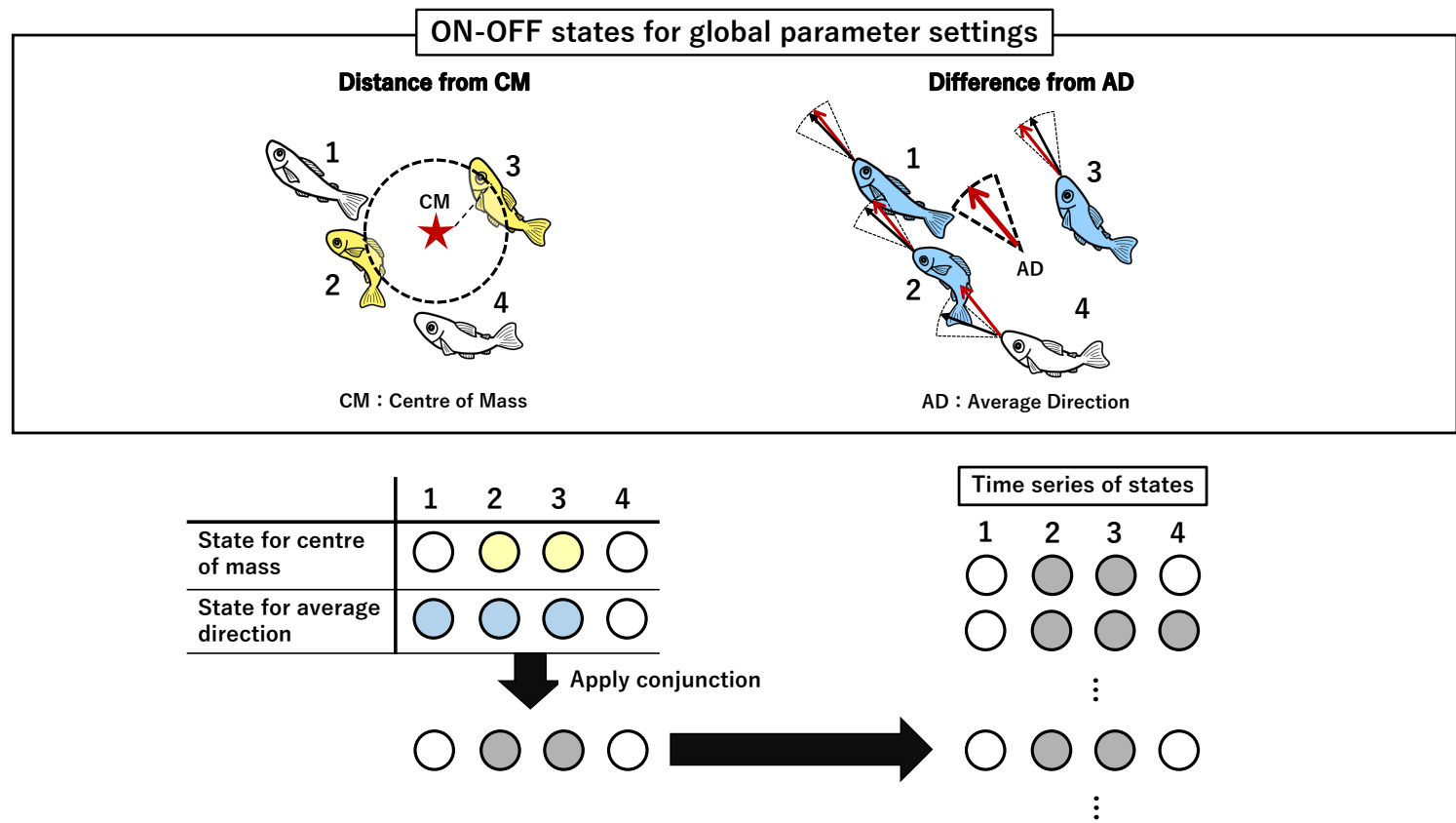

Figure 5. Definition of ON and OFF states for global parameter settings. Two parameters determine a school's state (Yellow: Centre of Mass and Blue: Average Direction). Coloured individuals are in the ON state. We calculate the conjunction of the two school states and obtain the final school state at time $t$. Subsequently, we compute $\Phi$ from a time series of these states by using PyPhi. We assume that the network structure is the same as that for the local parameter settings, i.e., the fully connected network without self-loop.

In this section, we examine the group integrity of two global parameters (Figure 5): the angle difference $\left(\Xi_{A D}\right)$ between each fish's direction and the average group direction at time $t$, and the distance $\left(\Xi_{C M}\right)$ between the position of a fish and the centre of mass of the group at time $t$. These two parameters $\left(\Xi_{A D}\right.$ and $\left.\Xi_{C M}\right)$ determine the collective states in this condition. The collective state at time $t$ in the global parameter settings is determined after calculating the conjunction (AND operator) of the two parameters as in the local parameter settings. Again, we compute TPM from a series of collective states with a fully connected network (no self-loop included) and obtain the corresponding $\Phi$ value to each collective state for the global parameters.

The two aforementioned averaged values are related to the measure of the collective behaviour domain. The average group direction is often used as the group polarity (i.e., the degree of alignment) $[4,15,29,78]$, whereas, the centre of mass is a useful measure for group clustering $[6,14,84-86]$. These interactions result in keeping a certain distance (direction) with the global state. For example, if a fish's distance from the centre of mass is always above its threshold $\left(\Xi_{C M}\right)$, then the fish cannot keep contact with the belonging group. If a fish's direction almost always differs from the average group direction $\left(\Xi_{A D}\right)$, then the fish again gradually diverts from the school to which it belongs. This is why the use of the AND operator makes sense for the collective state in the global parameter settings. If we change the AND connective to OR, then we cannot ensure this group coherence.

Note that there is a sharp difference between the local and global parameter settings in this concept. To refer to the average values, a priori is needed to assume that the group members have 
already been determined in advance. The average values for comparison contain the information of all the members at time $t$ despite their apparent pairwise interactions (because ON/OFF state is determined by the individual fish state and its global reference). In terms of a complex system, this global parameter setting works as feedback information from the entire school to each fish.

We can interpret the difference between the local and global parameters as follows. First, the collective state of the global settings works as a top-down system because the global reference can play the role of a feedback system to each member. As such, the group integrity is determined by the degree of connections with the central information (i.e., averaged values) through the continuous adjustments with the global reference. In contrast, the collective state of the local parameter settings works in a bottom-up approach because there is no assumption of the group in advance. Instead, the corrective state is determined as a building block through local interaction (this concept matches with the concept of Boid interaction). Both parameter settings represent typical ways of information processing (i.e., bottom-up and top-down), widely observed in the collective behaviour $[2,9,17,78]$.

Figure 6 shows some similarities and dissimilarities in the graph of the local parameter settings. In terms of the $\langle\Phi(N)\rangle$ values, both Figures 2 and 6 show an increase in $\langle\Phi(N)\rangle$ according to the group size. However, in terms of the relation of inter-distributions of heat maps, the discontinuity observed in Figure 2 is not seen in Figure 6 (Table S1). Instead, the discontinuity of $\langle\Phi(N)\rangle$ distributions in Figure 6 appears between two- and three-fish schools (Table S1: the matrix distance).

These observations imply that both the degree of the group integrities in a school highly as well as the discontinuity in the $\langle\Phi(N)\rangle$ distribution depend on the choice of the parameter settings. The local and global parameter settings suggest a different way that the agents recognise can their environment. The first is the interaction between each fish (the local parameter settings), and the other is the interaction at the global reference assuming that each fish in the group knows all the group members a priori (the global parameter settings). These contrasts in the interactions lead to differences in the integrity of their systems.
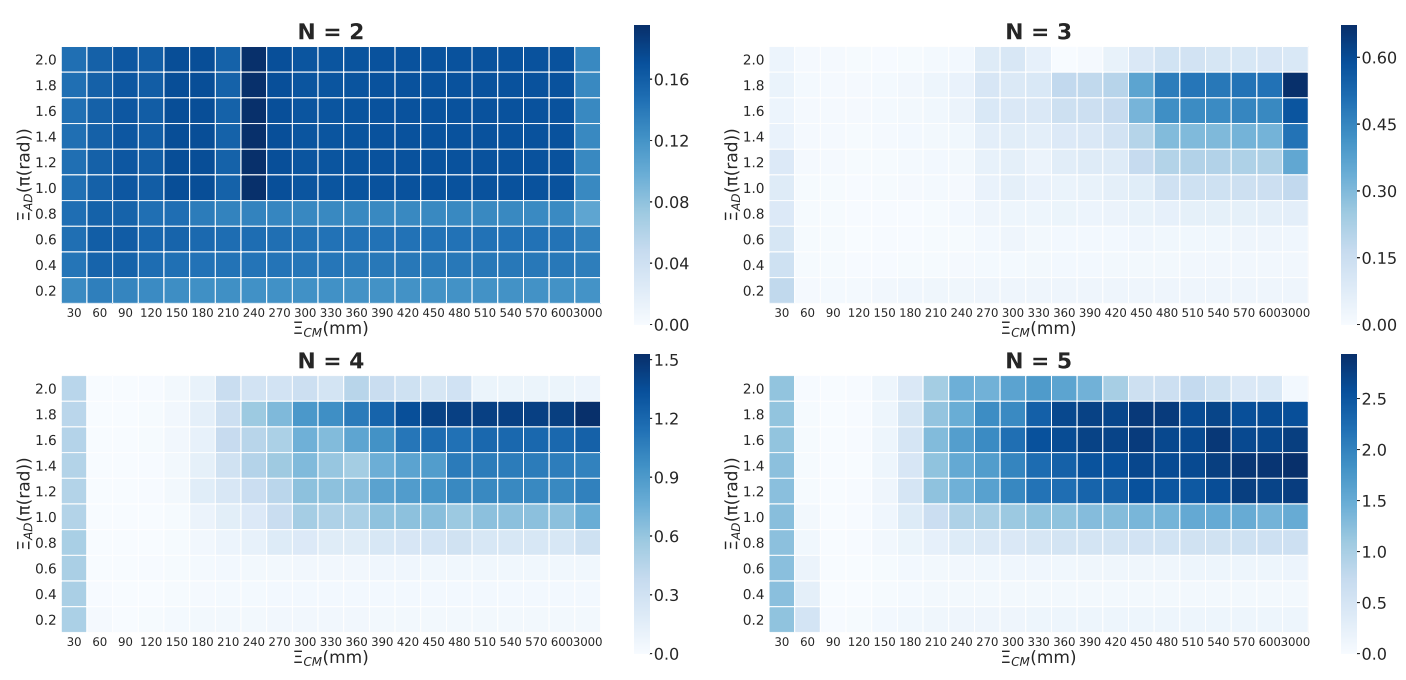

Figure 6. Heat maps on the global parameter settings for each group size ( $\Delta t=20 / 120 \mathrm{~s})$. We took the average of $\langle\Phi(N)\rangle$ for all datasets (colour bar). The horizontal axis shows the distance from the centre of mass $\left(\Xi_{C M}\right)$, and the vertical axis shows the difference from average direction $\left(\Xi_{A D}\right)$. All timescale figures are listed in Figure S5.

If the group integrities can contribute to their ways of forming aggregations, then it would be helpful to examine the power balance on $\langle\Phi(N)\rangle$ values between the local and global parameter settings. Figure 7 shows the comparison between the peak $\langle\Phi(N)\rangle$ values (top 20 for each) selected from the heat maps along with the timescales. As observed, the local and global parameter settings give qualitatively different $\langle\Phi(N)\rangle$ values. For clarity, we denote $\Phi_{\text {local }}$ (say, the local group integrity) 
for the local parameters and $\Phi_{\text {global }}$ (say, the global group integrity) for the global parameters. $\Phi_{\text {local }}$ is an intrinsic causal power generated by Boid-like local interactions, while the $\Phi_{\text {global }}$ is based on the interaction with the global reference. For a longer timescale, we previously confirmed that $\Phi(N)$ values for both parameter settings generally increase; however, they eventually saturate beyond a specific timescale. Figure 7 shows how each $\langle\Phi(N)\rangle$ value changes with timescales. The vertical axis represents the difference between $\Phi_{\text {local }}$ and $\Phi_{\text {global }}$, while the horizontal axis represents timescales. The difference between the two values gradually shifts from negative to positive. In other words, group integrities induced by global parameter settings (global group integrity) become dominant in the short timescales, while group integrities induced by local parameter settings (local group integrity) become dominant in the large timescales. Finally, the differences between the local and global group integrities diminish.

What does this mean to the fish's collective behaviour in this case? For the short timescale, the continuous adjustments among the fishes to their global reference are important for their integration, as the global group integrity increases when the position and directions of all the fishes are in a specific range with respect to their average information. However, in the long timescale, the local rather than the global group integrity is prioritised for the schools. The local group integrity tends to be lower in short time scales because the noisy movements of the fish weaken their predictabilities. This weak predictability reduces their causal connections. However, in short timescales, these causal connections of the global group integrity are relatively higher than that of the local group integrity because each fish shares the global information through their average values. The sharing of the same information sources can contribute to the increase in the causal connection among fishes.

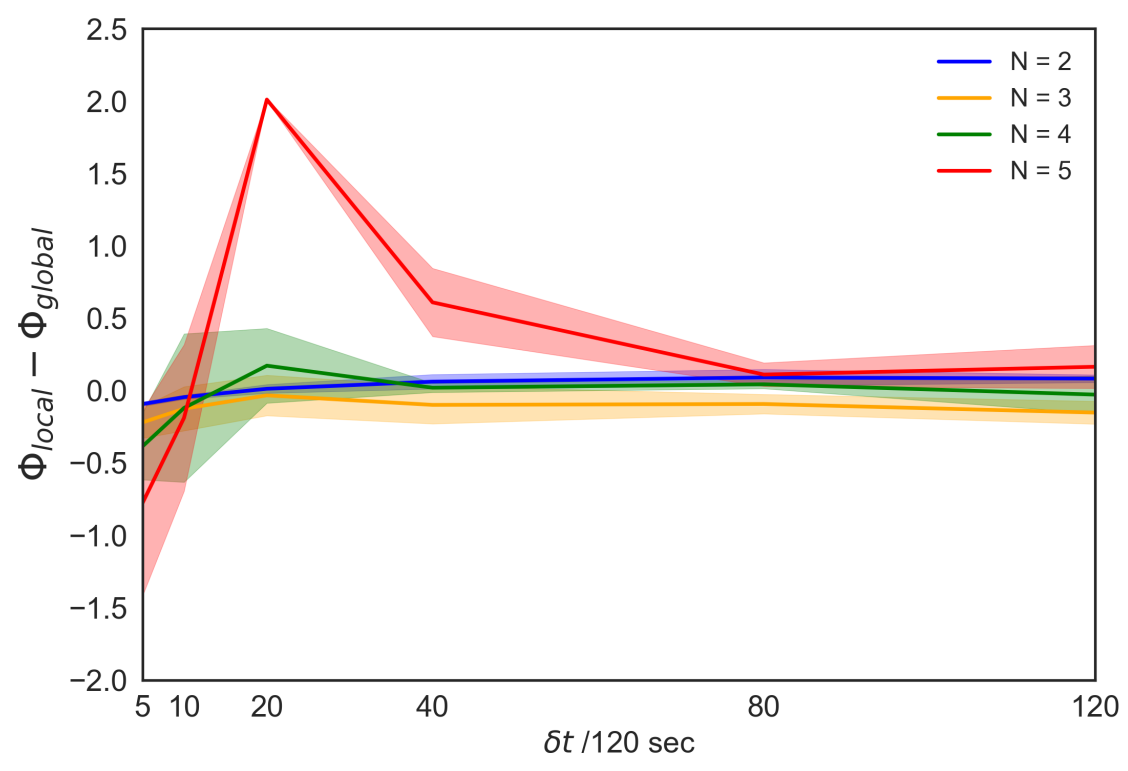

Figure 7. Difference between the average top $\langle\Phi(N)\rangle$ value of the local and global group integrities. The horizontal axis shows the timescales $(\Delta t=\delta t / 120$, where $\delta t=5,10,20,40,80,120)$. The negative and positive values on the vertical axis represent the global and local integrity dominance, respectively. The peak value of $N=5$ at $\Delta t=20 / 120$ is significantly larger than at the other peaks (Table 1 ).

We also confirm the outstanding property of a school of five fishes at $\Delta t=20 / 120 \mathrm{~s}$. Interestingly, this timescale approximately corresponds to the reaction time of the fishes [42]. The graph shows that a school of five fishes at $\Delta t=20 / 120 \mathrm{~s}$ is because of the dominance of the local group integrity compared with that in other school sizes (Table 1). Besides the local group integrity being based on Boid-like interactions, the peak shows that the Boid-like interactions completely take over the global interaction 
Table 1. Tukey-Kramer method for Figure 7 at $\Delta t=20 / 120$ (s). The notation, $D_{G-L}(\Phi(N))$, represents the difference between $\Phi_{\text {local }}(N)-\Phi_{\text {global }}(N)$ for group size $N$.

\begin{tabular}{|c||c|c|}
\hline & $D_{G-L}(\boldsymbol{\Phi}(\boldsymbol{N}))-D_{G-L}(\boldsymbol{\Phi}(\boldsymbol{M}))$ & p-value \\
\hline \hline$N=2-M=3$ & -0.049 & 0.999 \\
$N=2-M=4$ & 0.193 & 0.69 \\
$N=2-M=5$ & 2.31 & $<\mathbf{1 0}^{-7}$ \\
$N=3-M=4$ & 0.242 & 0.48 \\
$N=3-M=5$ & $\mathbf{2 . 3 6}$ & $<\mathbf{1 0}^{-8}$ \\
$N=4-M=5$ & $\mathbf{2 . 1 2}$ & $<\mathbf{1 0}^{-7}$ \\
\hline
\end{tabular}

with respect to the average values (Table 1). Note that no remarkable difference was observed among two-, three-, and four-fish schools. Only the school of five fishes showed strong local group integrity.

\subsection{Correlation between $\langle\Phi(N)\rangle$ and $\sigma^{2}(\Phi(N))$}

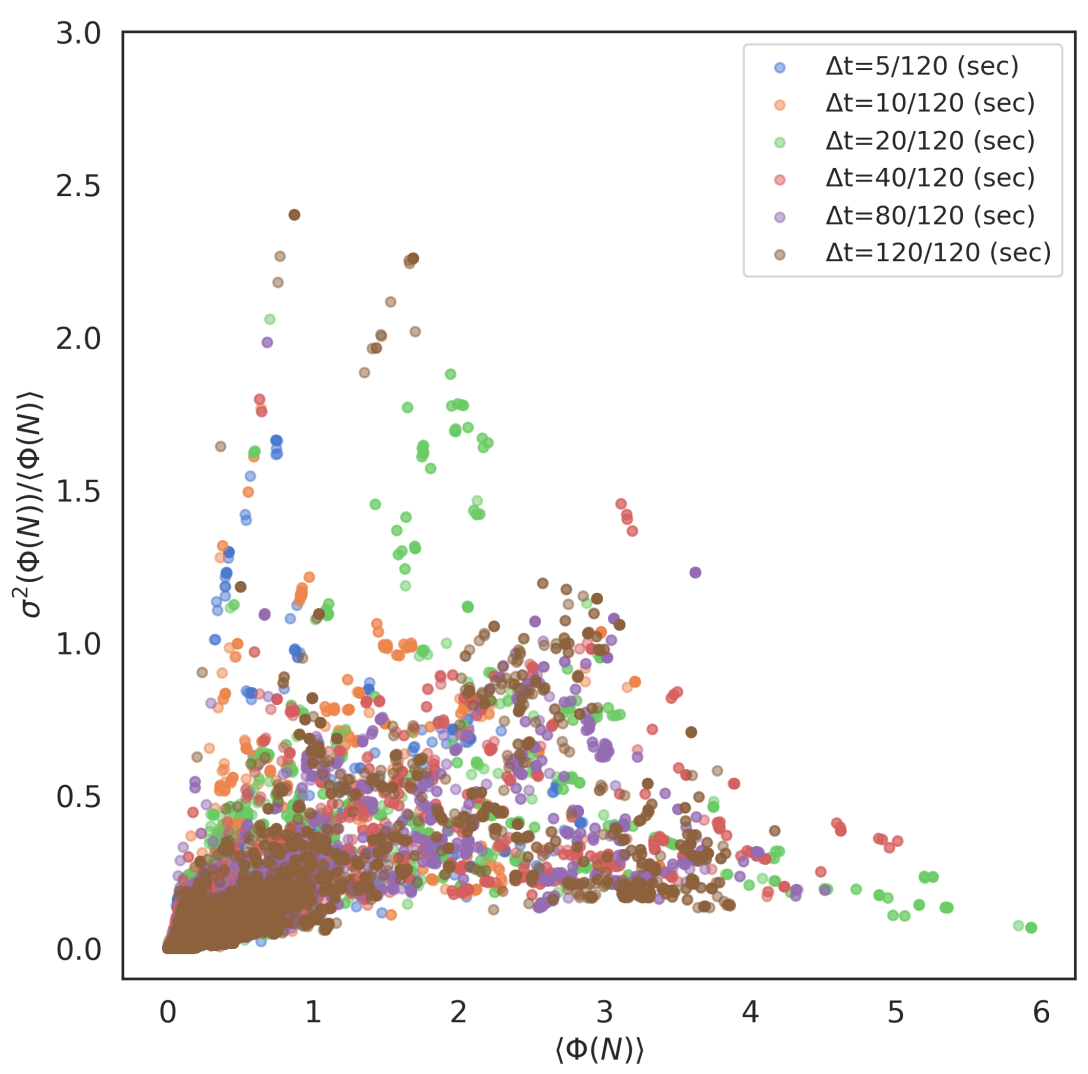

Figure 8. Correlation relation between $\langle\Phi(N)\rangle$ and its Fano scales, $\sigma^{2}(\Phi(N)) /\langle\Phi(N)\rangle$ (normalized variance) for the local parameter setting. Each colour corresponds to timescales. The correlation coefficients are $0.66(\Delta t=5 / 120 \mathrm{~s}), 0.71(\Delta t=10 / 120 \mathrm{~s}), 0.61(\Delta t=20 / 120 \mathrm{~s}), 0.72(\Delta t=40 / 120 \mathrm{~s})$, $0.75(\Delta t=80 / 120 \mathrm{~s})$, and $0.61(\Delta t=120 / 120 \mathrm{~s})$. For all the Pearson correlation tests, $p<10^{-30}$. For the global parameter settings, see Figure $S 6$.

It is worthy to note the positive correlation between the mean $\Phi$ values and its variance in a real school, especially in the local interaction systems. Figure 8 plots all $\langle\Phi(N)\rangle$ values and their Fano factors $\sigma^{2}(\Phi(N))$ (normalised variance) of all parameter settings. Those of the global parameter 
settings show the same result (Figure S6) but show a relatively weak tendency compared with the local systems. The strong correlation between $\langle\Phi(N)\rangle$ and its variance in real fish bears critical implications. This correlation suggests that the schools with large $\langle\Phi(N)\rangle$ values contain two extremes: small $\Phi(N)$ values and large $\Phi(N)$ values (recall each collective state has unique a $\Phi(N)$ value and $\langle\Phi(N)\rangle$ represents the average of those $2^{n} \Phi(N)$ values for $n$-fish school). The real school systems tend to have small $\Phi(N)$ values even if the group integrity (i.e., average $\Phi(N)$ values of all collective states) is significant. Note that the pure stochastic model, such as that generated using Markov process, never shows such a tendency (its variance is around 0.4 at most [77]). Their variances remain small even if the $\langle\Phi(N)\rangle$ increases. The high variances in a highly integrated system are one of the characteristic properties observed in a real school.

\subsection{New classification of schools as different autonomous systems}

As explained earlier, the aim of this study was to discriminate between the qualitatively different autonomous systems in terms of several parameter settings. Most studies on the collective behaviour rarely focus on small groups (as different systems from each other) and target information processing inside the groups ([51]).

The high group integrity regions (i.e., $\langle\Phi(N)\rangle$ values' cells) connect the group forming structure in terms of the internal causal structures, such as the leadership for the four-fish school. Here, we succeeded in classifying all schools consisting of two to five fishes and described four distinct autonomous systems (Figure 9). (I) The two-fish group shows followership relations in long timescales; (II) The three-fish group shows fission-fusion dynamics; and (III) the four-fish group has leadership as an autonomous system. Our analysis suggests that this autonomous system would have high intrinsic causal power owing to the presence of leadership. (IV) The five-fish group, in addition to leadership, shows dominating boid-like local interactions for long timescales. The intrinsic causal power of local interactions overwhelms the global interactions.

\begin{tabular}{|c|c|c|c|c|}
\hline & \multicolumn{2}{|c|}{ Short time scale } & \multicolumn{2}{|c|}{ Long time scale } \\
\hline & $\begin{array}{l}\text { Leader/ } \\
\text { Follower }\end{array}$ & $\begin{array}{l}\text { Local/ } \\
\text { Global }\end{array}$ & $\begin{array}{l}\text { Leader/ } \\
\text { Follower }\end{array}$ & $\begin{array}{l}\text { Local/ } \\
\text { Global }\end{array}$ \\
\hline $\mathrm{N}=2$ & $\mathrm{~N}$ & $\mathrm{~N}$ & $F$ & $N$ \\
\hline$N=3$ & $\mathrm{~N}$ & $\mathrm{~N}$ & $\mathrm{~N}$ & $N$ \\
\hline $\mathrm{N}=4$ & $L$ & $\mathrm{G}$ & $\mathrm{L}$ & $N$ \\
\hline $\mathrm{N}=5$ & $\mathrm{~L}$ & $\mathrm{G}$ & L & $\mathrm{L}$ \\
\hline
\end{tabular}

Figure 9. Classification of the schools. The first column classifies the existence of a leader (L), a follower $(\mathrm{F})$, or neither $(\mathrm{N})$. The second column classifies the three dominant interaction modes: local interaction $(\mathrm{L})$, global interaction $(\mathrm{G})$, or neither $(\mathrm{N})$. The same classification applies for short and long timescales.

(I) Two-fish school as "followership": the distribution of the school of two and three fishes has a wide similarity in the short timescales; however, this is not the case for longer timescales (Figure 9). The $\langle\Phi(N)\rangle$ values concentrated in the lower $\xi_{V F}$ parameters are as high as in the intact $\xi_{V F}$ parameters. The low $\xi_{V F}$ in the two-fish school shows one fish following the other within their narrow visual field. Recall that the MIP cuts always divide each other in the two-fish school. The MIP in the two-fish 
school suggests the existence of asymmetric information between them; this differs from the leadership relations. In particular, for the followership, if the same situation is reversed, it never generates the same $\langle\Phi(N)\rangle$ values (Figure 3 (b) and Figure 4 (b)). Therefore, we should discriminate the followership from the leadership.

(II) Three-fish school as "fission-fusion": Compared with other group size cases, this school can be characterised negatively. Unlike the two-fish school, there is no display of followership in this group size. Furthermore, there is no leadership relation as in the four- and five-fish schools and no local interaction dominance as in the five-fish school. The only confirmed fact observed in this study was that the three-fish school prefers to use the complete visual field (Figure 3 (a) and (b)). These parameter settings indicate that the collective states mainly depend on the distance. The relative distance is with respect to the group integrity of the three-fish school (i.e., $\langle\Phi(N)\rangle$ ). Therefore, the classification of "fission and fusion" seems to be suitable for the three-fish school.

(III) Four-fish school as "leadership": The leadership relation emerges only after the four-fish school. "Leadership" has been explained in detail in our previous study [77]. The selection of a fully connected network without a self-loop is based on our previous result because the MIP cut fits the group leader. Note the difference in leadership compared with preceding studies [42-44,49]. The derivation of the leadership is not based on the external group behaviour but on the internal causal structure. For example, the transfer entropy captures leadership as this school's behaviour but not as its autonomous system.

(IV) Five-fish schools as "Boid-like interaction": From our previous observation, only the five-fish school is able to show higher group integrities in the local parameter settings. Unlike the global parameter settings, the local parameter settings are defined as the bottom-up interactions. The group integrity in the global parameter settings tends to achieve high $\langle\Phi(N)\rangle$ values because each fish can access its global information. However, sharing the precise global information (example, average direction) becomes difficult when the size of the group increases. This difficulty seems to allow the local group integrity to overcome the global group integrity.

\section{Discussion}

The IIT analysis for the collective behaviour forces us to shift out focus from an external perspective (information transfer) to an internal perspective (internal causal structure). The former represents the events occurring in the system, while the latter represents the functions of the system $[51,57,69,70]$. Until now, the latter approach, from an internal perspective, barely applies to collective behaviour of animals. Transfer entropy is a representative method of the former approach, especially in a small group [42-44,49]. The transfer entropy approach first constructs by selecting a pair of individuals from the given group, then it measures the degree of information and misinformation [42], and finally determines the leadership relation in the group. From the external perspective, the leadership in the group is itself a ubiquitous property. All these approaches aimed to reveal the properties of the system behaviour of the group.

The importance of our approach is mainly based on two aspects: the shift from an external to an internal perspective, and a tentative answer to the question, "what is the criterion of the group?". Next, these aspects are discussed in detail.

First, the representative analysis from the external perspective is the transfer entropy analysis [42-44,49]. As discussed earlier, this method is constructed by selecting a pair of individuals from the group; however, this method only represents the predictability of each pair of individuals from their external behaviours in a series of events.

The analysis based on the internal perspective provides us with different perspectives because the collective behaviour from external perspectives is needless. The cellular automaton study in [51] could help in understanding this phenomenon. In this study, although IIT 3.0 can discriminate from Class I to Class IV, the cell number for their analysis was restricted to six. This number is significantly small to discriminate the external cell behaviour with respect to their corresponding rules. Therefore, there is 
no room here to determine the system by using the very little information of the cells behaviour. The discrimination becomes possible only if the cellular automaton's intrinsic causal structures of IIT 3.0 are considered but not their external behaviour.

The critical point is that the IIT-induced leadership corresponds to the positional leadership in the group without referring to the external behaviour. The correspondence with the IIT-induced leadership and external leadership (e.g., the positional leadership) is a posteori but not a priori. In fact, the transfer-entropy-induced leadership never generates the IIT-induced leadership. Our previous study indicates that the transfer entropy approach does not discriminate the leadership as an autonomous system. Therefore, the pairwise interaction analysis is not suitable for the autonomous system.

Until now, throughout our IIT analysis in this study, we defined two interactions: the local and global parameter settings. These interactions have qualitatively different meanings. The former represents the group integrity from the bottom-up perspective, while the latter represents the group integrity from the top-down perspective. We observed disagreements regarding the integrity between them, and this disagreement maximised at a certain timescale in the certain size of the group. In addition to the result of our previous study (i.e., Emergence of the leadership over the four-fish school), the timescale and new interaction are beneficial in understanding two new properties: the followership for the two-fish school and the local interaction dominance for the five-fish school. The fission-fusion property for the three-fish school can be defined negatively. Therefore, we can succeed to show the classification from the two- to five-fish schools, that is, the followership, fission-fusion dynamics, leadership, and Boid-like interaction. This is one of the most significant achievements of our study. Some studies have indicated the difference between two- and three-fish schools; however, their methods rarely discriminate a larger group size.

Furthermore, our IIT analysis led us to reconsider the fundamental question: "what is the criterion for forming a group?". This question is rarely asked but simply assumed. How? The information transfer in the group, for instance, assumes the individual's collective behaviour as one collection [38,42]. The other example can be seen in the criticality arguments $[14,15,78]$. The analysis of the scale-free correlation in the birds can only be possible when we define the average values (for example mean directions or speeds). The computation of these average values implicitly contains the decision group's criterion of the observer because we assume the given data set already forms the group.

We do not insist that their assumption on the group's criterion is itself inappropriate. Rather, this assumption seems inevitable for group analysis. A sufficient number of individuals is likely to satisfy those criteria. However, in the case of a small group similar to the transition from two- to five-fish school, this problem is not negligible because there is still no consensus on the group criterion for such sizes. The question of what kind of the difference exists among these small groups has remained untouched up to the present.

We consider that the heap paradox would help to shape the core of this problem [30]. The heap paradox is constructed by adding each grain on the floor sequentially when the aggregation of grains becomes a heap. The key of this paradox is in the fact that the same successive procedure (i.e. grain dropping) can make the system different, qualitatively. The paradox asks us when does qualitative difference begin and how can we know that. This paradox can be observed all over the domain of complex systems concerning with parts and whole relations (e.g., Neurons-build consciousness $[56,57,60,61]$ and individuals' build-group behaviour $[27,34,87])$. The paradox overlaps our arguments of collective behaviour on the issue: "the criterion of the group behaviour".

The clue for understanding the heap paradox is in the way the grain is distributed. To understand this, it is sufficient to confirm that the random distribution of the grain never constructs the heap; rather, the heap is constructed by the distribution of flat grain. In other words, the agent doing the same procedure inevitably commits his/her decision on where to drop the grain, anticipating the forthcoming unbuilt heap. The heap paradox implicitly includes the agent's commitment to grain droppings. The paradox begins when we forget these agent's ontological commitments. 
The agent's commitment can be considered in the context of a manipulative view in IIT 3.0. As Tononi claims, "something can be said to exist only if it has cause-effect power"[61]; the causal-effect structure of an autonomous system represents "what causes what", considering all counterfactual states. Lombardi et al. correctly pointed out that the central idea of "differences that make differences" represents the potential intervention (manipulation) in the cause-effect structure of the system [71]. We can also apply this argument to collective behaviour. The intrinsic causal structure in a school represents how each fish virtually affects one another to maintain an aggregation. The manipulative view in IIT 3.0 involves the agent's commitment to the intrinsic causal perspective.

Now we are ready to give the two tentative answers to the paradox from IIT 3.0: from the inner-system perspective and the inter-system perspective.

Figure 8 corresponds to the former answer. As we have discussed earlier, the strong correlation between $\langle\Phi(N)\rangle$ and Fano factor $\sigma^{2}(\Phi(N)) /\langle\Phi(N)\rangle$ is innate for a real school (no observation in the artificial condition, such as the random Markov networks.) This correlation indicates that the high average group integrity in a real school has both low and high $\Phi$ values, simultaneously. In other words, the real school group coexists in tightly mutual connected with feedback (united: high $\Phi$ ) and weak connections with no feedback (separated: low $\Phi$ ) individuals exist in the same fish school. Unlike other methods, the ability of multiple $\Phi(N)$ values in the same group is critical in IIT 3.0. This coexistence in IIT 3.0 can prevent the paradox's assumption because a law that excluded the middle (i.e. any proposition must be true or true for its negation: in this case, we mean that the grains would be united or not) becomes invalid. This is one possible answer to the paradox.

The latter answer (inter-system) can be obtained from the classification of schools as autonomous systems. Our finding is that the difference in group size, differs the autonomous system. This classification itself makes the paradox's assumption invalid because the properties of the group change according to the group's size from IIT 3.0 perspective. There is no definitive point where the group behaviour begins, but its definition may be different for the way of forming the group in a given environment. In other words, the question of when the school begin becomes invalid. This is another possible answer to the paradox.

We investigated the schools. By applying IIT 3.0, there are possibilities for new approaches to the problem of the collective behaviour which remains unsolved. We believe that our approach sheds light on not only the collective behaviour but also the application of IIT 3.0.

\section{Materials and Methods}

\subsection{Ethics statement}

This study was carried out in strict accordance with the recommendations in the Guide for the Care and Use of Laboratory Animals of the National Institutes of Health. The protocol was approved by the Committee on the Ethics of Animal Experiments of the University of Tsukuba (Permit Number: 14-386). All efforts were made to minimise suffering.

\section{2. $\Phi$ computation}

All computations, in this study, were performed using the PyPhi software package with the CUT_ONE_APPROXIMATION to $\Phi$.

\subsection{Experimental Settings}

We studied ayus (Plecoglossus altivelis), also known as sweetfish, which are common and widely bred in Japan. Juvenile ayus (approximately 7-14 cm in body length) display typical schooling behaviour, though adult ayus tend to show territorial behaviour in environments where fish density is low. We purchased fingerlings from Tarumiyoushoku (Kasumigaura, Ibaraki, Japan) and housed them in a controlled laboratory. Approximately 150 fish lived in a $0.8 \mathrm{~m}^{3}$ tank of continuously filtered and recycled fresh water with a temperature maintained at $16.4^{\circ} \mathrm{C}$. They were fed with commercial food 
pellets. Just before each experiment was conducted, fish were randomly chosen and separated to form a school of each size and were moved to an experimental arena without pre-training. The experimental arena consisted of a $3 \times 3 \mathrm{~m}^{2}$ shallow white tank. The water depth was approximately $15 \mathrm{~cm}$ so that schools would be approximately 2D. The fish were recorded with an overhead grey-scale video camera (Library GE 60; Library Co. Ltd., Tokyo, Japan) at a spatial resolution of $640 \times 480$ pixels and a temporal resolution of $120 \mathrm{fps}$.

\subsection{Data Summary}

Table 2. Data Summary. $N$ : Number of individuals (Unit: None), $x$ : Average distance (Unit: (mm)), $v$ : Average velocity (Unit: (mm) per second), Error (S.D.) (Unit: Degrees (rad)), $d_{\text {min }}$ : Minimum distance (Unit: (mm)), Total Time Steps (steps)

\begin{tabular}{|c|c|c|c|c|c|}
\hline$N$ & Average distance $(\mathrm{mm})$ & Average velocity $(\mathrm{mm} / \mathrm{s})$ & Error (S.D.) & Minimum distance (mm) & Total Time Steps \\
\hline \multirow{3}{*}{2} & 166.3 & 268.8 & 0.18 & 1.90 & 106961 \\
\hline & 90.67 & 271.68 & 0.23 & 0.10 & 99431 \\
\hline & 122.0 & 256.08 & 0.18 & 1.60 & 107206 \\
\hline \multirow{4}{*}{3} & 170.8 & 301.2 & 0.23 & 1.80 & 90051 \\
\hline & 159.1 & 343.2 & 0.14 & 1.83 & 83654 \\
\hline & 173.1 & 300.0 & 0.13 & 2.82 & 97446 \\
\hline & 132.0 & 240.0 & 0.19 & 1.67 & 93931 \\
\hline \multirow{3}{*}{4} & 164.3 & 270.72 & 0.14 & 1.18 & 106327 \\
\hline & 141.5 & 190.8 & 0.12 & 1.38 & 103226 \\
\hline & 114.9 & 148.56 & 0.38 & 1.83 & 98126 \\
\hline \multirow{3}{*}{5} & 143.8 & 259.92 & 0.28 & 0.79 & 102895 \\
\hline & 146.0 & 213.12 & 0.12 & 1.16 & 97346 \\
\hline & 143.7 & 259.2 & 0.28 & 1.44 & 92116 \\
\hline
\end{tabular}

\subsection{Timescales}

We prepared the trajectory data in different time intervals (In Python $\boldsymbol{X}[:: \delta t]$ ) and applied smoothing by convolving three data points to reduce noise. $\delta t$ is a timescale in $\delta t / 120$.

\subsection{The definition of ON and OFF state for each parameter}

We define a function for each parameter that returns either $0(\mathrm{OFF})$ or $1(\mathrm{ON})$, for any given input value. Generally, we denote a function as $F_{i}^{t}(\cdot)$, where $F$ is the name of the function, $i$ is the index of the individual, and $t$ is the time. The arguments of the function can be either in the position vectors, $x_{i}(t)$, or the velocity vectors, $v_{i}(t)$, of each individual, at time $t$. In general, the dimensions of these vectors are $d \leq 3$; the experimental setup used here gives $d=2$, and the number of individuals is $N$.

\subsubsection{Local parameters}

- Distance function $D_{i}^{t}\left(x_{1}(t), x_{2}(t), \cdots, x_{n}(t)\right): \mathbb{R}^{d} \times \mathbb{R}^{d} \times \cdots \times \mathbb{R}^{d} \rightarrow\{0,1\}$

For each individual, $i$, we obtain a set $S_{i}^{t}=\left\{j \mid d\left(x_{i}(t), x_{j}(t)\right)<\xi_{D}, j \neq i\right\}$ of all other individuals within a specified distance, $\xi_{D}$. Here $d(\boldsymbol{x}, \boldsymbol{y})$ gives the Euclidean distance between $\boldsymbol{x}$ and $\boldsymbol{y}$. Then, $D_{i}^{t}\left(x_{1}(t), x_{2}(t), \ldots, x_{n}(t)\right)=1$ when $\left|S_{i}^{t}\right|>0$ and is 0 otherwise, where $|S|$ denotes the number of elements of the set, $S$.

- Visual field function $B_{i}^{t}\left(x_{1}(t), x_{2}(t), \ldots, x_{n}(t), \boldsymbol{v}_{1}(t), \boldsymbol{v}_{2}(t), \cdots, \boldsymbol{v}_{n}(t)\right): \mathbb{R}^{d} \times \mathbb{R}^{d} \times \cdots \times \mathbb{R}^{d} \rightarrow$ $\{0,1\}$

For each individual we form the set $O_{i}^{t}=\left\{j \mid \arg \left(v_{i}(t), x_{i}(t)-x_{j}(t)\right)<\xi_{V F}, j \neq i\right\}$ of all other individuals whose velocity vectors point in a direction within an angle $\xi_{V F}$ of the focal 
individual. The function $\arg \left(x_{1}(t), x_{2}(t)\right)$ gives the angle between the two vectors. Then, $B_{i}^{t}\left(x_{1}(t), x_{2}(t), \ldots, x_{n}(t), v_{1}(t), v_{2}(t), \cdots, v_{n}(t)\right)=1$ when $\left|O_{i}^{t}\right|>0$ and is 0 otherwise.

- Turning rate function $T_{i}^{t}\left(\boldsymbol{v}_{i}(t), \boldsymbol{v}_{i}(t-\Delta t)\right): \mathbb{R}^{d} \times \mathbb{R}^{d} \rightarrow\{0,1\}$

The turning rate function returns 1 when an individual's turning rate exceeds a specified threshold, $\delta$. That is, $T_{i}^{t}\left(\boldsymbol{v}_{i}(t), \boldsymbol{v}_{i}(t-\Delta t)\right)=1$ when $\arg \left(\boldsymbol{v}_{i}(t), \boldsymbol{v}_{i}(t-\Delta t)\right) \geq \xi_{\mathrm{TR}}$ and is 0 otherwise. The time step used in this study is from $\Delta t=0.05$ to $\Delta t=1.0 \mathrm{~s}$.

To obtain the states of the school, we take a conjunction of this result, that is, $D_{i}^{t}\left(x_{1}(t), x_{2}(t), \cdots, x_{n}(t)\right) \wedge B_{i}^{t}\left(v_{1}(t), v_{2}(t), \cdots, v_{n}(t)\right) \wedge T_{i}^{t}\left(\boldsymbol{v}_{i}(t), \boldsymbol{v}_{i}(t-\Delta t)\right)$ for each individual, $i$. The conjunction is given as $\wedge:\{0,1\}^{2} \rightarrow\{0,1\}$ where $1 \wedge 1=1$ and is 0 otherwise. Thus the state of each individual $i$ at time $t$ is $s_{i}\left(t ; \xi_{D}, \xi_{V F}, \xi_{T R}\right) \in\{0,1\}$ which depends on the three parameter values $\left(\xi_{D}, \xi_{V F}, \xi_{T R}\right)$. The state of the school at time $t$ is a vector $s(t)=\left(s_{1}(t), s_{2}(t), \ldots, s_{n}(t)\right) \in\{0,1\}^{n}$, where the parameter dependence has been omitted for simplicity.

\subsubsection{Global parameters}

- Average direction function $A v d_{i}^{t}\left(\boldsymbol{V}(t), \boldsymbol{v}_{i}(t)\right): \mathbb{R}^{d} \times \mathbb{R}^{d} \rightarrow\{0,1\}$

$\boldsymbol{V}(t)$ is the average of $\left\{\boldsymbol{v}_{1}(t), \boldsymbol{v}_{2}(t), \ldots, \boldsymbol{v}_{n}(t)\right\}$. If an individual's direction of motion deviates from the average by more than a threshold amount, $\Xi_{A D}$, then the individual is in the OFF state: that is, $A v d_{i}^{t}\left(\boldsymbol{V}(t), \boldsymbol{v}_{i}(t)\right)=1$ when $\arg \left(\boldsymbol{V}(t), \boldsymbol{v}_{i}(t)\right) \leq \Xi_{A D}$, and is 0 otherwise.

- Centre of mass function $\operatorname{Com}_{i}^{t}\left(\boldsymbol{X}(t), \boldsymbol{x}_{i}(t)\right): \mathbb{R}^{d} \times \mathbb{R}^{d} \rightarrow\{0,1\}$

$\boldsymbol{X}(t)$ is the average of $\left\{x_{1}(t), x_{2}(t), \cdots, x_{n}(t)\right\}$. If an individual is further from $X(t)$ than a specified threshold $\Xi_{C M}$ then the individual is in the OFF state: that is, $\operatorname{Com}_{i}^{t}\left(\boldsymbol{X}(t), \boldsymbol{x}_{i}(t)\right)=1$ when $d\left(\boldsymbol{X}(t), \boldsymbol{x}_{i}(t)\right) \leq \Xi_{C M}$ and is 0 otherwise.

To obtain the state of the school, we take a conjunction of these results to obtain a state for each individual, which depends on the pair $\left(\Xi_{A D}, \Xi_{C M}\right):, s_{i}\left(t ; \Xi_{A D}, \Xi_{C M}\right)=A v d_{i}^{t}\left(\boldsymbol{V}(t), \boldsymbol{v}_{i}(t)\right) \wedge$ $\operatorname{Com}_{i}^{t}\left(\boldsymbol{X}(t), \boldsymbol{x}_{i}(t)\right) \in\{0,1\}$. The state of the school at time $t$ is then a vector $s(t)=$ $\left(s_{1}(t), s_{2}(t), \ldots, s_{n}(t)\right) \in\{0,1\}^{n}$, where the parameter dependence has been omitted for simplicity.

Supplementary Materials: The following are available at http://www.mdpi.com//xx/1/5/s1, Figure S1: $\langle\Phi(N)\rangle$ for all timescales, http://www.mdpi.com//xx/1/5/s2, Figure S2: $\sigma^{2}(\Phi(N))$ for all timescales,http: / /www.mdpi.com//xx/1/5/s3, Figure S3: The box plot of Figure 3 (ii) where $\xi_{T R} \geq 0.05$. http://www.mdpi. com//xx/1/5/s4, Figure S4: The box plot of Figure 4 (ii) where $\xi_{T R} \geq 0.05$. http://www.mdpi.com//xx/1/5/s5, Figure S5: $\langle\Phi(N)\rangle$ for all timescales for the global parameter settings. http://www.mdpi.com//xx/1/5/s6, Figure S6: The correlation between $\langle\Phi(N)\rangle$ and $\sigma^{2}(\Phi(N)) /\langle\Phi(N)\rangle$ for the global parameter settings. Table S1: All statistical tests on Figure 3, Figure 4, Figure 7 and Figure 8 are included.

Author Contributions: Conceptualisation, T.N., K.S. and Y.M; methodology, T.N. and K.S.; software, T.N. and K.S.; validation, T.N. and K.S. ; formal analysis, T.N. and K.S.; investigation, T.N. and K.S.; resources, T.N., T.H, T.F; data curation, T.N. and K.S.; writing-original draft preparation, T.N., K.S. and Y.M; writing-review and editing, T.N., T.H, Y.,M, H.M, T.T., T.F; visualisation, T.N. and K.S.

Funding: This research received no external funding.

Conflicts of Interest: The authors declare no competing interests.

\section{Abbreviations}

The following abbreviations are used in this manuscript:

IIT Integrated Information Theory

SOC Self-organising Criticality 


\section{References}

1. Buhl, J.; Sumpter, D.; Couzin, I.; Hale, J.; Despland, E.; Miller, E.; Simpson, S. From Disorder to Order in Marching Locusts. Science 2006, 312, 1402-1406. doi:10.1126/science.1125142.

2. Yates, C.; Erban, R.; Escudero, C.; Couzin, I.; Buhl, J. Inherent noise can facilitate coherence in collective swarm motion. PNAS 2009, 106, 5464-5469. doi:https: / / doi.org/10.1126/science.1125142.

3. Bazazi, S.; Bartumeus, F.; Hale, J.J.; Couzin, I.D. Intermittent motion in desert locusts: Behavioural complexity in simple environments. PLoS Computational Biology 2012. doi:10.1371/journal.pcbi.1002498.

4. Attanasi, A.; Cavagna, A.; Castello, D.; Giardina, I.; Melillo, S.; Parisi, L.; Pohl, O.; Rossaro, R.; Shen, E.; Silvestri, E.; Viale, M. Finite-size scaling as a way to probe near-criticality in natural swarms. Phys. Rev. Lett 2014, 113, 238102. doi:https://doi.org/10.1103/PhysRevLett.113.238102.

5. Attanasi, A.; Cavagna, A.; Del Castello, L.; Giardina, I.; Melillo, S.; Parisi, L.; Pohl, O.; Rossaro, B.; Shen, E.; Silvestri, E.; Viale, M. Collective Behaviour without Collective Order in Wild Swarms of Midges. PLoS Computational Biology 2014, 10, [1307.5631]. doi:10.1371/journal.pcbi.1003697.

6. Murakami, H.; Tomaru, T.; Nishiyama, Y.; Moriyama, T.; Niizato, T.; Gunji, Y.P. Emergent runaway into an avoidance area in a swarm of soldier crabs. PLoS ONE 2014. doi:10.1371/journal.pone.0097870.

7. Ioannou, C.C.; Guttal, V.; Couzin, I.D. Predatory fish select for coordinated collective motion in virtual prey. Science 2012, [arXiv:arXiv:astro-ph/0005074v1]. doi:10.1126/science.1218919.

8. Strandburg-Peshkin, A.; Twomey, C.R.; Bode, N.W.; Kao, A.B.; Katz, Y.; Ioannou, C.C.; Rosenthal, S.B.; Torney, C.J.; Wu, H.S.; Levin, S.A.; Couzin, I.D. Visual sensory networks and effective information transfer in animal groups. Current Biology 2013. doi:10.1016/j.cub.2013.07.059.

9. Berdahl, A.; Torney, C.J.; Ioannou, C.C.; Faria, J.J.; Couzin, I.D. Emergent sensing of complex environments by mobile animal groups. Science 2013. doi:10.1126/science.1225883.

10. Murakami, H.; Niizato, T.; Tomaru, T.; Nishiyama, Y.; Gunji, Y.P. Inherent noise appears as a Lévy walk in fish schools. Scientific Reports 2015. doi:10.1038/srep10605.

11. Niizato, T.; Murakami, H.; Sangu, K.; Tomaru, T.; Sonoda, K.; Nishiyama, Y.; Gunji, Y.P. Local perspectives of Plecoglossusaltivelis determine searching strategy. AIP Conference Proceedings, 2017. doi:10.1063/1.4992536.

12. Ballerini, M.; Cabibbo, N.; Candelier, R.; Cavagna, A.; Cisbani, E.; Giardina, I.; Orlandi, A.; Parisi, G.; Procaccini, A.; Viale, M.; Zdravkovic, V. Empirical investigation of starling flocks: a benchmark study in collective animal behaviour. Animal Behaviour 2008, [0802.1667]. doi:10.1016/j.anbehav.2008.02.004.

13. Cavagna, A.; Cimarelli, A.; Giardina, I.; Parisi, G.; Santagati, R.; Stefanini, F.; Viale, M. Scale-free correlations in starling flocks. Proceedings of the National Academy of Sciences of the United States of America 2010, [0911.4393]. doi:10.1073/pnas.1005766107.

14. Cavagna, A.; Duarte Queirós, S.M.; Giardina, I.; Stefanini, F.; Viale, M. Diffusion of individual birds in starling flocks. Proceedings of the Royal Society B: Biological Sciences 2013, [1206.4434]. doi:10.1016/S0022-5347(17)41086-X.

15. Bialek, W.; Cavagna, A.; Giardina, I.; Mora, T.; Pohl, O.; Silvestri, E.; Viale, M.; Walczak, A.M. Social interactions dominate speed control in poising natural flocks near criticality. Proceedings of the National Academy of Sciences 2014, [1307.5563]. doi:10.1073/pnas.1324045111.

16. Attanasi, A.; Cavagna, A.; Del Castello, L.; Giardina, I.; Jelic, A.; Melillo, S.; Parisi, L.; Pohl, O.; Shen, E.; Viale, M. Emergence of collective changes in travel direction of starling flocks from individual birds' fluctuations. Journal of the Royal Society Interface 2015, [1410.3330]. doi:10.1098/rsif.2015.0319.

17. Mora, T.; Walczak, A.M.; Del Castello, L.; Ginelli, F.; Melillo, S.; Parisi, L.; Viale, M.; Cavagna, A.; Giardina, I. Local equilibrium in bird flocks. Nature Physics 2016, [1511.01958]. doi:10.1038/nphys3846.

18. Tsuchiya, M.; Giuliani, A.; Hashimoto, M.; Erenpreisa, J.; Yoshikawa, K. Emergent Self-Organized Criticality in Gene Expression Dynamics: Temporal Development of Global Phase Transition Revealed in a Cancer Cell Line. PLOS ONE 2015, 10, 1-33. doi:10.1371/journal.pone.0128565.

19. Phillips, J. Fractals and self-organized criticality in proteins. Physica A: Statistical Mechanics and its Applications 2014, 415, 440 - 448. doi:10.1016/j.physa.2014.08.034.

20. Rosenblatt, F. The Perceptron: A Probabilistic Model for Information Storage and Organization in The Brain. Psychological Review 1958, pp. 65-386. doi:10.1.1.335.3398. 
21. Hopfield, J.J. Neural networks and physical systems with emergent collective computational abilities. Proceedings of the National Academy of Sciences of the United States of America 1982, 79, 2554-2558. doi:10.1073/pnas.79.8.2554.

22. Hesse, J.; Gross, T. Self-organized criticality as a fundamental property of neural systems. Frontiers in Systems Neuroscience 2014, 8, 166. doi:10.3389/fnsys.2014.00166.

23. Lynn, C.W.; Bassett, D.S. The physics of brain network structure, function and control. Nature Reviews Physics 2018, 1, 318-332. doi:10.1038/s42254-019-0040-8.

24. Franks, N.R.; Dornhaus, A.; Fitzsimmons, J.P.; Stevens, M. Speed versus accuracy in collective decision making. Proceedings of the Royal Society B: Biological Sciences 2003. doi:10.1098/rspb.2003.2527.

25. Dyer, J.R.; Johansson, A.; Helbing, D.; Couzin, I.D.; Krause, J. Leadership, consensus decision making and collective behaviour in humans. Philosophical Transactions of the Royal Society B: Biological Sciences 2009. doi:10.1098/rstb.2008.0233.

26. Bose, T.; Reina, A.; Marshall, J.A. Collective decision-making, 2017. doi:10.1016/j.cobeha.2017.03.004.

27. Couzin, I. Collective minds. Nature 2007. doi:10.1038/445715a.

28. Couzin, I.D.; Ioannou, C.C.; Demirel, G.; Gross, T.; Torney, C.J.; Hartnett, A.; Conradt, L.; Levin, S.A.; Leonard, N.E. Uninformed individuals promote democratic consensus in animal groups. Science 2011. doi:10.1126/science.1210280.

29. Pinkoviezky, I.; Couzin, I.D.; Gov, N.S. Collective conflict resolution in groups on the move. Physical Review E 2018. doi:10.1103/PhysRevE.97.032304.

30. Bak, P.; Tang, C.; Wiesenfeld, K. Self-organized criticality. Physical Review A 1988, [arXiv:1011.1669v3]. doi:10.1103/PhysRevA.38.364.

31. Tetzlaff, C.; Okujeni, S.; Egert, U.; Wörgötter, F.; Butz, M. Self-organized criticality in developing neuronal networks. PLoS Computational Biology 2010. doi:10.1371/journal.pcbi.1001013.

32. Niizato, T.; Gunji, Y.P. Fluctuation-driven flocking movement in three dimensions and scale-free correlation. PLoS ONE 2012. doi:10.1371/journal.pone.0035615.

33. Gunji, Y.P. Self-organized criticality in asynchronously tuned elementary cellular automata. Complex Systems 2014, [1306.4419]. doi:10.1142/9789814602136_0021.

34. Gunji, Y.P.; Sakiyama, T.; Murakami, H. Punctuated equilibrium based on a locally ambiguous niche. BioSystems 2014. doi:10.1016/j.biosystems.2014.05.005.

35. Niizato, T.; Murakami, H. Entangled time in flocking : Multi-time-scale interaction reveals emergence of inherent noise. PLoS ONE 2018, 13, 1-21. doi:10.1371/journal.pone.0195988.

36. Herbert-Read, J.E.; Krause, S.; Morrell, L.J.; Schaerf, T.M.; Krause, J.; Ward, A.J. The role of individuality in collective group movement. Proceedings of the Royal Society B: Biological Sciences 2013. doi:10.1098/rspb.2012.2564.

37. Jolles, J.W.; Boogert, N.J.; Sridhar, V.H.; Couzin, I.D.; Manica, A. Consistent Individual Differences Drive Collective Behavior and Group Functioning of Schooling Fish. Current Biology 2017, [1601.08202]. doi:10.1016/j.cub.2017.08.004.

38. Katz, Y.; Tunstrom, K.; Ioannou, C.C.; Huepe, C.; Couzin, I.D. Inferring the structure and dynamics of interactions in schooling fish. Proceedings of the National Academy of Sciences 2011, [arXiv:1604.05974v2]. doi:10.1073/pnas.1107583108.

39. Gautrais, J.; Ginelli, F.; Fournier, R.; Blanco, S.; Soria, M.; Chaté, H.; Theraulaz, G. Deciphering Interactions in Moving Animal Groups. PLoS Computational Biology 2012. doi:10.1371/journal.pcbi.1002678.

40. Staniek, M.; Lehnertz, K. Symbolic transfer entropy. Physical Review Letters 2008, [1007.0357v1]. doi:10.1103/PhysRevLett.100.158101.

41. Butail, S.; Mwaffo, V.; Porfiri, M. Model-free information-theoretic approach to infer leadership in pairs of zebrafish. Physical Review E 2016, [arXiv:1011.1669v3]. doi:10.1103/PhysRevE.93.042411.

42. Crosato, E.; Jiang, L.; Lecheval, V.; Lizier, J.T.; Wang, X.R.; Tichit, P.; Theraulaz, G.; Prokopenko, M. Informative and misinformative interactions in a school of fish, 2018, [1705.01213]. doi:10.1007/s11721-018-0157-x.

43. Wang, X.R.; Miller, J.M.; Lizier, J.T.; Prokopenko, M.; Rossi, L.F. Quantifying and tracing information cascades in swarms. PLoS ONE 2012. doi:10.1371/journal.pone.0040084. 
44. Wang, X.R.; Miller, J.M.; Lizier, J.T.; Prokopenko, M.; Rossi, L.F. Measuring information storage and transfer in swarms. Proceedings of the Eleventh European Conference on the Synthesis and Simulation of Living Systems (ECAL 2011) 2011.

45. Lizier, J.T.; Prokopenko, M.; Zomaya, A.Y. Local information transfer as a spatiotemporal filter for complex systems. Physical Review E - Statistical, Nonlinear, and Soft Matter Physics 2008, [0809.3275]. doi:10.1103/PhysRevE.77.026110.

46. Lizier, J.T.; Prokopenko, M.; Zomaya, A.Y. Local measures of information storage in complex distributed computation. Information Sciences 2012. doi:10.1016/j.ins.2012.04.016.

47. Sun, J.; Bollt, E.M. Causation entropy identifies indirect influences, dominance of neighbors and anticipatory couplings. Physica D: Nonlinear Phenomena 2014, [1504.03769]. doi:10.1016/j.physd.2013.07.001.

48. James, R.G.; Barnett, N.; Crutchfield, J.P. Information Flows? A Critique of Transfer Entropies. Physical Review Letters 2016, [1512.06479]. doi:10.1103/PhysRevLett.116.238701.

49. Tomaru, T.; Murakami, H.; Niizato, T.; Nishiyama, Y.; Sonoda, K.; Moriyama, T.; Gunji, Y.P. Information transfer in a swarm of soldier crabs. Artificial Life and Robotics 2016. doi:10.1007/s10015-016-0272-y.

50. Yagmur Erten, E.; Lizier, J.T.; Piraveenan, M.; Prokopenko, M. Criticality and information dynamics in epidemiological models. Entropy 2017. doi:10.3390/e19050194.

51. Albantakis, L.; Tononi, G. The intrinsic cause-effect power of discrete dynamical systems-from elementary cellular automata to adapting animats. Entropy 2015. doi:10.3390/e17085472.

52. Tononi, G. Information measures for conscious experience. Arch Ital Biol 2001, 4, 367-371. doi:10.1186/1471-2202-4-31.

53. Tononi, G.; Sporns, O. Measuring information integration. BMC Neuroscience 2003. doi:10.1186/1471-2202-4-31.

54. Barrett, A.B.; Seth, A.K. Practical measures of integrated information for time- series data. PLoS Computational Biology 2011. doi:10.1371/journal.pcbi.1001052.

55. Balduzzi, D.; Tononi, G. Qualia: The geometry of integrated information. PLoS Computational Biology 2009, [NIHMS150003]. doi:10.1371/journal.pcbi.1000462.

56. Tononi, G. An Integrated iNformation Theory of Consciousness. In Encyclopedia of Consciousness; 2010; [1606.06737]. doi:10.1016/B978-012373873-8.00014-1.

57. Oizumi, M.; Albantakis, L.; Tononi, G. From the Phenomenology to the Mechanisms of Consciousness: Integrated Information Theory 3.0. PLoS Computational Biology 2014, [arXiv:1011.1669v3]. doi:10.1371/journal.pcbi.1003588.

58. Oizumi, M.; Tsuchiya, N.; Amari, S.i. A unified framework for information integration based on information geometry 2015. 113, [1510.04455]. doi:10.1073/pnas.1603583113.

59. Oizumi, M.; Amari, S.I.; Yanagawa, T.; Fujii, N.; Tsuchiya, N. Measuring Integrated Information from the Decoding Perspective. PLoS Computational Biology 2016, [1505.04368]. doi:10.1371/journal.pcbi.1004654.

60. Tononi, G.; Koch, C. Consciousness: Here, there and everywhere?, 2015, [1405.7089]. doi:10.1098/rstb.2014.0167.

61. Tononi, G.; Boly, M.; Massimini, M.; Koch, C. Integrated information theory: From consciousness to its physical substrate, 2016. doi:10.1038/nrn.2016.44.

62. Mayner, W.; Marshall, W.; Albantakis, L.; Findlay, G.; Marchman, R.; Tononi, G. PyPhi: A toolbox for integrated information theory. PLoS Computational Biology 2018. doi:10.1371/journal.pcbi.1006343.

63. Massimini, M.; Ferrarelli, F.; Huber, R.; Esser, S.K.; Singh, H.; Tononi, G. Neuroscience: Breakdown of cortical effective connectivity during sleep. Science 2005, [20]. doi:10.1126/science.1117256.

64. Alkire, M.T.; Hudetz, A.G.; Tononi, G. Consciousness and anesthesia. Science (New York, N.Y.) 2008. doi:10.1126/science.1149213.Consciousness.

65. Gosseries, O.; Di, H.; Laureys, S.; Boly, M. Measuring Consciousness in Severely Damaged Brains. Annual Review of Neuroscience 2014. doi:10.1146/annurev-neuro-062012-170339.

66. Khajehabdollahi, S.; Abeyasinghe, P.; Owen, A.; Soddu, A. The emergence of integrated information, complexity, and consciousness at criticality. bioRxiv 2019, [https:/ / www.biorxiv.org/content/early/2019/01/15/521567.full.pdf]. doi:10.1101/521567.

67. Mediano, Pedro A.M., F.J.C.; Shanahan, M. Integrated Information and Metastability in Systems of Coupled Oscillators 2016. [arXiv:1606.08313]. 
68. Mori, H.; Oizumi, M. Information integration in a globally coupled chaotic system. The 2018 Conference on Artificial Life: A Hybrid of the European Conference on Artificial Life (ECAL) and the International Conference on the Synthesis and Simulation of Living Systems (ALIFE) 2018, pp. 384-385. doi:https://doi.org/10.1162/isal_a_00074.

69. Albantakis, L.; Marshall, W.; Hoel, E.; Tononi, G. What caused what? A quantitative account of actual causation using dynamical causal networks. Entropy 2019, [1708.06716]. doi:10.3390/e21050459.

70. Marshall, W.; Kim, H.; Walker, S.I.; Tononi, G.; Albantakis, L. How causal analysis can reveal autonomy in models of biological systems. Philosophical Transactions of the Royal Society A: Mathematical, Physical and Engineering Sciences 2017, [1708.07880]. doi:10.1098/rsta.2016.0358.

71. Lombardi, O.; López, C. What Does 'Information' Mean in Integrated Information Theory? Entropy 2018, 20. doi:10.3390/e20120894.

72. Wolfram, S. Statistical mechanics of cellular automata. Reviews of Modern Physics 1983. doi:10.1103/RevModPhys.55.601.

73. Wolfram, S. Universality and complexity in cellular automata. Physica D: Nonlinear Phenomena 1984. doi:10.1016/0167-2789(84)90245-8.

74. Langton, C.G. Computation at the edge of chaos: Phase transitions and emergent computation. Physica D: Nonlinear Phenomena 1990. doi:10.1016/0167-2789(90)90064-V.

75. Wuensche, A.; Lesser, M. The global dynamics of cellular automata : an atlas of basin of attraction fields of one-dimensional cellular automata; 1992.

76. Gunji, Y.P. Inducing Class 4 Behavior on the Basis of Lattice Analysis. Complex Systems 2010. doi:10.25088/complexsystems.19.2.177.

77. Niizato, T.; Sakamoto, K.; Mototake, Y.; Murakami, H.; Tomaru, T.; Hoshika, T.; Fukushima, T. Finding continuity and discontinuity in fish schools via integrated information theory. PLOS ONE 2020, 15, 1-29, [1812.00718]. doi:10.1371/journal. pone.0229573.

78. Cavagna, A.; Cimarelli, A.; of the ..., G.I. Scale-free correlations in starling flocks. Proceedings of the ... 2010.

79. Couzin, I.; Krause, J.; James, R.; of theoretical ..., R.G. Collective memory and spatial sorting in animal groups. Journal of theoretical ... 2002.

80. Vicsek, T.; Zafeiris, A. Collective motion, 2012, [1010.5017]. doi:10.1016/j.physrep.2012.03.004.

81. Sonoda, K.; Kitamura, E.S.; Tani, I.; Shirakawa, T.; Gunji, Y.P. Analyzing double image illusion through double indiscernibility and lattice theory. TripleC 2011.

82. Hildenbrandt, H.; Carere, C.; Hemelrijk, C.K. Self-organized aerial displays of thousands of starlings: A model. Behavioral Ecology 2010. doi:10.1093/beheco/arq149.

83. Hemelrijk, C.K.; Hildenbrandt, H. Scale-Free Correlations, Influential Neighbours and Speed Control in Flocks of Birds. Journal of Statistical Physics 2014. doi:10.1007/s10955-014-1154-0.

84. Miller, N.; Gerlai, R. Redefining membership in animal groups. Behavior Research Methods 2011. doi:10.3758/s13428-011-0090-z.

85. Quera, V.; Beltran, F.S.; Givoni, I.E.; Dolado, R. Determining shoal membership using affinity propagation. Behavioural Brain Research 2013. doi:10.1016/j.bbr.2012.11.031.

86. Viscido, S.V.; Shrestha, S. Using quantitative methods of determining group membership to draw biological conclusions. Animal Behaviour 2015. doi:10.1016/j.anbehav.2015.03.007.

87. Olfati-Saber, R.; Fax, J.A.; Murray, R.M. Consensus and cooperation in networked multi-agent systems. Proceedings of the IEEE 2007, [1009.6050]. doi:10.1109/JPROC.2006.887293. 